Nat. Hazards Earth Syst. Sci., 17, 905-924, 2017

https://doi.org/10.5194/nhess-17-905-2017

(C) Author(s) 2017. This work is distributed under

the Creative Commons Attribution 3.0 License.

\title{
On the resonance hypothesis of storm surge and surf beat run-up
}

\author{
Nazmi Postacioglu ${ }^{1}$, M. Sinan Özeren ${ }^{2}$, and Umut Canlı ${ }^{1}$ \\ ${ }^{1}$ Department of Physics, Istanbul Technical University, Maslak 34469, Istanbul, Turkey \\ ${ }^{2}$ Eurasia Institute of Earth Sciences, Istanbul Technical University, Maslak 34469, Istanbul, Turkey \\ Correspondence to: M. Sinan Özeren (ozerens@itu.edu.tr)
}

Received: 13 October 2016 - Discussion started: 1 November 2016

Revised: 11 May 2017 - Accepted: 12 May 2017 - Published: 19 June 2017

\begin{abstract}
Resonance has recently been proposed as the fundamental underlying mechanism that shapes the amplification in coastal run-up for storm surges and surf beats, which are long-wavelength disturbances created by fluid velocity differences between the wave groups and the regions outside the wave groups. It is without doubt that the resonance plays a role in run-up phenomena of various kinds; however, we think that the extent to which it plays its role has not been completely understood. For incident waves, which we assume to be linear, the best approach to investigate the role played by the resonance would be to calculate the normal modes by taking radiation damping into account and then testing how those modes are excited by the incident waves. Such modes diverge offshore, but they can still be used to calculate the run-up. There are a small number of previous works that attempt to calculate the resonant frequencies, but they do not relate the amplitudes of the normal modes to those of the incident wave. This is because, by not including radiation damping, they automatically induce a resonance that leads to infinite amplitudes, thus preventing them from predicting the exact contribution of the resonance to coastal run-up. In this study we consider two different coastal geometries: an infinitely wide beach with a constant slope connecting to a flat-bottomed deep ocean and a bay with sloping bottom, again, connected to a deep ocean. For the fully 1-D problem we find significant resonance if the bathymetric discontinuity is large. The linearisation of the seaward boundary condition leads to slightly smaller run-ups. For the 2-D ocean case the analysis shows that the wave confinement is very effective when the bay is narrow. The bay aspect ratio is the determining factor for the radiation damping. One reason why we include a bathymetric discontinuity is to mimic some natural settings where bays and gulfs may lead to abrupt depth gradients such as the Tokyo Bay. The other reason is, as men-
\end{abstract}

tioned above, to test the role played by the depth discontinuity for resonance.

\section{Introduction}

During the last decades, several analytical and numerical studies of coastal run-up were published (see Synolakis, 1987; Brocchini, 1998; Brocchini and Gentile, 2001; Carrrier et al., 2003; Kânoğlu, 2005; Kânoğlu and Synokalis, 2005; Antuono and Brocchini, 2008; Özeren and Postacioglu, 2012; Stefanakis et al., 2015), most of which made use of Carrier-Greenspan transformations (Carrrier et al., 2003). Some of these works, for instance Stefanakis et al. (2015, 2011) and Ezersky et al. (2013b), identified the resonance as the fundamental factor for the run-up amplification. The bulk of the present study will be dedicated to determining the physical settings in which this might be the case.

In the past, several researchers looked at resonance aspect of the coastal run-up. Among those, the ones that are the most relevant to the discussion in the present study are Stefanakis et al. (2015), Stefanakis et al. (2011), Carrier and Noiseux (1983), Ezersky et al. (2013a), Fuentes et al. (2015), Volker et al. (2010), and Yamazaki and Cheung (2011). The last two of these studies report coastal resonance mechanisms leading to amplified run-ups during the 2009 Samoa and 2010 Chile tsunamis respectively. During the 1970s questions arose as to whether the presence of beaches in the vicinity of ports contribute to port resonances through surf beats. One interesting work whose geometric setting is somewhat similar to ours is that of Bowers (1977). However, this work looked at a flat-bottomed bay rather than a sloping bay that acted as the resonator. Additionally, it lacked a semi-infinite ocean, and it instead connected to a wider channel. But more im- 
portantly, Bowers considered only the incident waves having wavelengths greater than the width of the wider channel, prohibiting more than one mode radiating offshore. The work by Carrier and Noiseux (1983) does not explicitly mention resonance, but their formulation clearly shows a constructive interference by multiple reflections of an obliquely incident tsunami wave for a particular set of incidence angles. Some of the studies looked at the resonant mechanism in experimental settings (Ezersky et al., 2013a; Abcah et al., 2016).

In our modelling, we will be considering monochromatic incident waves, simply because this makes it easier to shed light on the resonance. However, the mathematical algorithm we will develop is not limited to monochromatic waves but is capable of calculating run-up for any kind of offshore source including the earthquakes, submarine landslides or atmospheric pressure perturbations.

This article deals with the transient run-up response of a sloping channel (or bay) to an incident wave. Here, the term transient is used for the wave evolution before the standing wave regime sets in. As such, the problem presents itself as an initial value problem. Such an initial value problem can be difficult and expensive to handle by purely numerical approaches. The reason for the difficulty is that, on the offshore boundary, it is difficult to make a distinction between the incident and reflected waves (for a detailed discussion, see Antuono and Brocchini, 2007, who use the method of characteristics where separate expressions for the incident and reflected waves do exist). One way of surmounting this difficulty numerically is to take a computational domain so large that, by the time the reflected waves arrive at the offshore boundary, the standing wave regime would have set in the coastal region. For instance, Stefanakis et al. (2011) did one-dimensional numerical simulations to understand the run-up amplification by non-leading long waves. They cast the problem as a boundary value problem and imposed an offshore boundary condition, at distance $L^{\prime}$ from the undisturbed shoreline, for the free surface elevation as $\eta^{\prime}= \pm 2 \eta_{0}^{\prime I} \sin \left(\omega^{\prime} t^{\prime}\right)$, where $t^{\prime}$ is time. However, this model, as will be explained in detail later, tends to overestimate the run-up if the open boundary lies in one of the nodes of the standing wave that will eventually set in. When the incident and reflected wave distinction is made (see, for example, Antuono and Brocchini, 2010), the run-up amplification factor (defined as $r^{\prime} /\left(2 \eta_{0}^{\prime I}\right)$, where $\eta_{0}^{\prime I}$ is the incident wave amplitude and $r^{\prime}$ is the run-up) remains finite as long as the frequency of the incident wave is real. This has independently been shown in Antuono and Brocchini (2010).

Our purpose in the present work is to determine the way in which the free modes near the coast are excited by the incident waves by taking the radiation damping into account. We will examine resonance in two different geometric settings: first in a 1-D slope which connects to a 1-D channel with a flat bottom and then, again, in a 1-D slope that connects to a semi-infinite 2-D ocean with a flat bathymetry (see
Fig. 1). When the wavelength of the incident wave is much shorter than the width of the sloping channel, the completely 1-D model is a good approximation of the natural case and we can neglect the geometric spreading of the waves at the toe of the sloping bay (this geometry will be referred to as Model-1 during the rest of the article). If this is not the case, then a 2-D model (Model-2) near the mouth of the bay becomes necessary (see Fig. 1). The Coriolis acceleration is neglected in both cases as we limit ourselves to small scales. Storm surges and meteotsunamis can excite Kelvin waves in the coastal areas, and such waves travelling along the coast can come across bay mouths and force the normal modes of the bay, so the analysis here is not confined to one specific type of wave.

Model-1 is actually solvable, through fast Fourier transforms (see Ezersky et al., 2013b), without necessarily resorting to the coastal free modes. However, generalising this solution approach to 2-D in the deep ocean part is computationally very expensive, requiring a solution of an integral equation for each frequency component to calculate the transient response. Hence, the real importance of the technique developed in this manuscript becomes more apparent in Model2 , which can be of great engineering importance in modelling coastal amplifications of storm surges in places like the Tokyo Bay, which has a sloping bathymetry (Kataoka et al., 2013).

\section{Basic equations}

Model-1 consists of a channel of constant slope $\alpha$ and length $L^{\prime}$ that connects to a another channel of uniform depth $\alpha L^{\prime}+$ $D^{\prime}$ (see Fig. 1). Parameter $D^{\prime}$ is the discontinuity in depth at the toe of the slope. The maximum depth of the sloping channel is $\alpha L^{\prime}$.

The governing equations we shall use over the sloping part of the geometry are nonlinear shallow water equations:

$\partial_{t^{\prime}} u^{\prime}+u^{\prime} \partial_{x^{\prime}} u^{\prime}+g \partial_{x^{\prime}} \eta^{\prime}=0$,
$\eta_{t^{\prime}}^{\prime}+\partial_{x^{\prime}}\left(\left(\alpha x^{\prime}+\eta^{\prime}\right) u^{\prime}\right)=0$,

where $u^{\prime}$ is depth-averaged velocity in offshore-pointing $x$ direction, $t^{\prime}$ is time, $g^{\prime}$ is the acceleration due to gravity and $\eta^{\prime}$ is the free surface elevation. For the flat part of the domain, for Model-1, the linearised versions of the same equations,

$\partial_{t^{\prime}} u^{\prime}+g \partial_{x^{\prime}} \eta^{\prime}=0$,

$\eta_{t^{\prime}}^{\prime}+\partial_{x^{\prime}}\left(\left(\alpha L^{\prime}+D^{\prime}\right) u^{\prime}\right)=0$,

are used. Note that, when we eventually start to discuss Model-2, we will generalize Eqs. (3) and (4) into two dimensions. Let us now define non-dimensional quantities as

$$
\begin{aligned}
& x=x^{\prime} / L^{\prime}, \quad \eta=\eta^{\prime} /\left(\alpha L^{\prime}\right) \\
& t=t^{\prime} \sqrt{\alpha g^{\prime} / L^{\prime}}, \quad u=u^{\prime} / \sqrt{g^{\prime} \alpha L^{\prime}}, \quad D=D^{\prime} /\left(\alpha L^{\prime}\right) .
\end{aligned}
$$




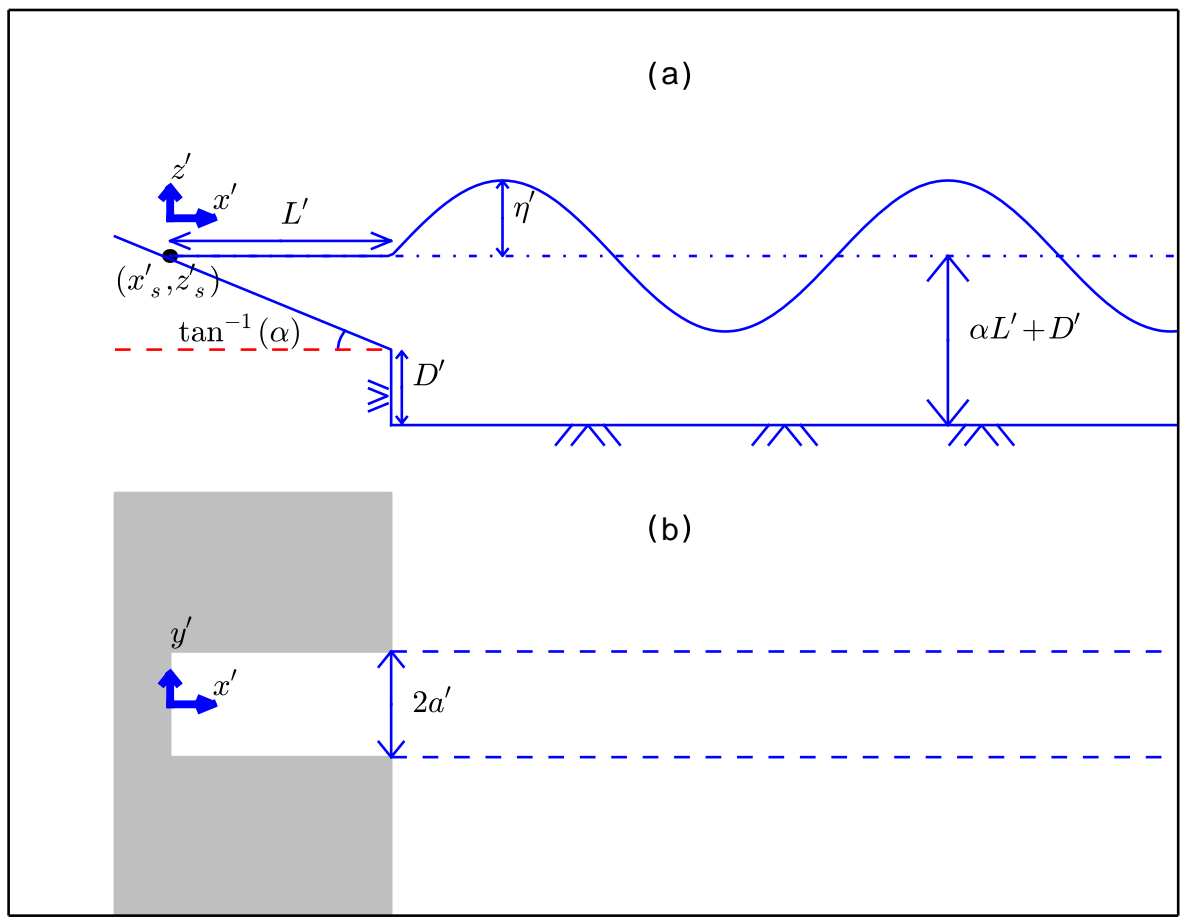

Figure 1. Panel (a) is the side view of the incident wave at instant $t^{\prime}=0$. The black dot is the shoreline, and $\left(x_{s}^{\prime}, z_{s}^{\prime}\right)$ are its coordinates, with $z_{s}^{\prime}$ being equal to run-up $r^{\prime}$. Panel (b) is the geometry of the channel seen from above. In Model-1 the sloping channel is connected to a deeper channel of the same width, see broken lines in panel (b). In Model-2 the channel opens to a semi-infinite ocean. The width of the channel is $2 a^{\prime}$. Non-dimensional quantities are defined as $x=x^{\prime} / L^{\prime}, y=y^{\prime} / L^{\prime}, z=z^{\prime} /\left(\alpha L^{\prime}\right), \eta=\eta^{\prime} /\left(\alpha L^{\prime}\right), D=D^{\prime} /(\alpha L)$ and $a=a^{\prime} / L^{\prime}$.

A hodograph transformation introduced by Carrrier et al. (2003), also called Carrier-Greenspan transformation (hereafter referred to as CG), uses "distorted time" $\lambda$ and a potential $\varphi$ defined as

$\lambda=t-u$

with

$u=-\partial_{\sigma} \varphi /(2 \sigma), \quad \eta=\partial_{\lambda} \varphi-\left(\partial_{\sigma} \varphi\right)^{2} /\left(8 \sigma^{2}\right)$,

where

$\sigma=\sqrt{x+\eta}$

The nonlinear shallow water equations accordingly become (Carrrier et al., 2003)

$\partial_{\lambda \lambda}^{2} \varphi-\frac{1}{4 \sigma} \partial_{\sigma} \varphi-\frac{\partial_{\sigma \sigma}^{2} \varphi}{4}=0$

To treat the incident wave problem, the initial conditions everywhere are

$\eta(t=0, x)=\eta_{\text {initial }}^{I}$

and

$u(t=0, x)=\frac{-\eta_{\text {initial }}^{I}}{\sqrt{D+1}}$ for the free surface elevation $\eta$ and the fluid velocity $u$ respectively. We assume that both quantities are initially zero over the slope $(0<x<1)$. The minus sign in Eq. (11) is due to the fact that the progressive wave advances in the negative $x$ direction towards the coast on the left.

\section{Green's function and the free mode expansion}

For the flat part of the domain $(x>1)$ we propose the following solution

$$
\begin{aligned}
& \eta(t, x>1)=\int_{-\infty}^{\infty}\left(\widetilde{\eta}^{I}(\omega) \exp \left(i \omega\left(t+\frac{x-1}{\sqrt{D+1}}\right)\right)\right. \\
& \left.+R(\omega) \exp \left(i \omega\left(t-\frac{x-1}{\sqrt{D+1}}\right)\right)\right) \mathrm{d} \omega,
\end{aligned}
$$

where $\widetilde{\eta}^{I}(\omega)$ and $R(\omega)$ are the temporal Fourier transforms of the incident and the reflected waves (to be calculated) respectively. Here, $\widetilde{\eta}_{\omega}^{I}$ is defined as

$\tilde{\eta}^{I}(\omega)=\frac{1}{2 \pi} \int_{-\infty}^{\infty} \eta^{I}(t, x=1) \exp (-i \omega t) \mathrm{d} t$.

The integrand in the right-hand side of Eq. (13), for all times, can be inferred from the initial condition as $\eta^{I}(t, x=1)=$ 
$\eta^{I}(0,1+t \sqrt{D+1})$. Note that the choice of the point $x=1$ is completely arbitrary. Let us now propose a solution over the slope as

$\varphi=\int_{-\infty}^{\infty} A_{0}(\omega) J_{0}(2 \omega \sigma) \exp (i \omega \lambda) \mathrm{d} \omega$

where $A_{0}(\omega)$ is the amplitude of the wave over the slope and $J_{0}$ is the Bessel function of the first kind of order zero.

The corresponding free surface elevation near $x=1^{-}$(toe of the slope) is then given as

$\eta(t, x)=\int_{-\infty}^{\infty} i \omega A_{0}(\omega) J_{0}(2 \omega \sqrt{x}) \exp (i \omega t) \mathrm{d} \omega$.

Note that we performed a linearisation here by taking $\lambda=t$ and $\sigma=\sqrt{x}$, because we assume that in the deeper part of the domain, waves are small. The unknown coefficients $R(\omega)$ and $A_{0}(\omega)$ are to be determined from the continuity conditions at the toe of the slope (see Sect. A1 for the matrix form of these two equations). The zero subscript for $A$ is used because for Model-1, having a fully one-dimensional geometry, only one coefficient is needed. Later, other coefficients will also be needed when we consider two spatial dimensions.

The linearised free surface and flux continuity conditions at the toe $(x=1)$ are used to obtain $A_{0}(\omega)$ and $R(\omega)$ as

$$
\begin{aligned}
& A_{0}(\omega)=\frac{2 \sqrt{D+1}}{\omega\left(i \sqrt{D+1} J_{0}(2 \omega)-J_{1}(2 \omega)\right)} \widetilde{\eta}^{I}(\omega), \\
& R(\omega)= \\
& \quad\left(-1+\frac{2 \sqrt{D+1}}{\sqrt{D+1} J_{0}(2 \omega)+i J_{1}(2 \omega)} J_{0}(2 \omega)\right) \widetilde{\eta}^{I}(\omega) .
\end{aligned}
$$

Here, by linear boundary condition at $x=1$ we mean that $x=\sigma^{2}, \lambda=t$ and depth is equal to $x$ rather than $x+\eta$.

Both $A_{0}(\omega)$ and $R(\omega)$ have simple poles at $\omega=0$. The non-vanishing poles are on the upper complex plane due to the conservation of energy through the radiation damping, because, on these poles, the ratio $A_{0}(\omega) / \widetilde{\eta}^{I}(\omega)$ and $R(\omega) / \widetilde{\eta}^{I}(\omega)$ both diverge and the wave can sustain itself without an incident wave and is fuelled solely by the initial conditions (see Longuet-Higgins, 1967 for a similar radiation damping problem over a circular submerged sill); these are called the free modes. Note that the conservation of energy requires that the amplitude of the incident wave is equal to that of the reflected wave with $\left|\widetilde{\eta}^{I}(\omega)\right|=|R(\omega)|$ when the frequency is real. However, this condition is relaxed when the frequency is no longer real because the energy density averaged over one cycle of oscillation evolves in time. See also Synolakis (1988) for rigorous proof that the frequencies of the free modes are indeed on the upper complex half-plane for $D=0$. Our argument based on the conservation of energy is more general and can be applied to any bathymetric profile.
Recently Stefanakis et al. (2015) attempted to calculate resonant frequencies for a geometry similar to Ezersky et al. (2013b) but imposed an offshore Dirichlet condition of the form $\eta=2 \eta_{0} \sin (\omega t)$ at a specified point $x=L$ using a numerical wave maker. With this boundary condition their results diverged at certain discrete real frequencies (Eq. 46 in Stefanakis et al., 2015). They attributed this to run-up resonance. However, one can always adjust the frequencies in such a way that the reflected wave and the incident wave are off-phase by $\pi$ at $x=L$, therefore leading to a destructive interference, requiring an infinite run-up yielding to such a finite oscillation at $x=L$. There is no simple relation between the amplitude of oscillation at a specified point and the amplitude of the incident wave unless the incident and reflected waves are in phase at this particular point. Therefore, relating the ratio between $\eta_{0}$ and the run-up to the resonance is meaningless. This problem can partially be remedied by imposing an artificial relaxation zone (Stefanakis et al., 2015; Madsen and Schäffer, 2010). In this work, we replace this approach with a physically realistic initial value problem where our definition of resonance is based on the ratio between the run-up and the amplitude of the incident wave. Not only will the resonant frequencies we calculate will be complex, but their real parts will also be substantially higher than those found by Stefanakis et al., 2015 when the discontinuity, $D$, is small (see Table 1). Only in the limit of large $D$ do the complex roots of the denominator $\left(i \sqrt{D+1} J_{0}(2 \omega)-J_{1}(2 \omega)\right)$ of $A_{0}(\omega)$ approach those of Dirichlet condition $J_{0}(2 \omega)=0$. Note that all of the resonant frequencies that we calculate in this work are independent of any nonlinearity. The reason for this is that we linearise the boundary conditions at the toe of the slope. The modes themselves $\left(J_{0}(2 \omega \sigma)\right)$, on the other hand, are affected by shoaling nonlinearity. Note that by the frequencies of the modes, we mean the frequency of the oscillation at the toe of the slope $(x=1)$. In Sect. 4.2 we will show that for higher modes the shoaling nonlinearities are dominant over those created by the boundary condition at the toe.

Now consider an incident wave of the following form:

$\eta_{0}^{I}(t, x)=\delta\left(t-t_{0}+\frac{(x-1)}{\sqrt{D+1}}\right)$,

where $\delta$ is the Dirac delta function. The zero index of $t$ in Eq. (18) relates to the phase of the incident wave. The response, $\varphi$, to such Dirac-type incident wave will be called Green's function $G\left(\lambda, t_{0}, \sigma\right)$. Remember that $A_{0}(\omega) J_{0}(2 \omega \sigma)$ is the temporal Fourier transform of $\varphi$. In Eq. (16) if we replace $\widetilde{\eta}^{I}$ by $\exp \left(-i \omega t_{0}\right) / 2 \pi$, then $A_{0}(\omega) J_{0}(2 \omega \sigma)$ will become the Fourier transform of Green's function. This Green's function in Fourier domain is given as

$$
\begin{aligned}
& \widetilde{G}\left(\omega, t_{0}, \sigma\right)= \\
& \quad \frac{\sqrt{D+1} \exp \left(-i \omega t_{0}\right)}{\pi \omega\left(i \sqrt{D+1} J_{0}(2 \omega)-J_{1}(2 \omega)\right)} J_{0}(2 \omega \sigma) .
\end{aligned}
$$


Table 1. The complex natural frequencies multiplied by 2 using both the Müller method and the asymptotic approach (see Eq. A5) for Model-1 are tabulated. Note that $2 \omega_{k}$ tend to the roots of $J_{0}$ for large $D$. All nonlinearities are neglected in calculating these frequencies.

\begin{tabular}{lrrrrr}
\hline & $2 \omega_{1}$ & $2 \omega_{2}$ & $2 \omega_{3}$ & $2 \omega_{4}$ & $2 \omega_{5}$ \\
\hline (Müller) $D=0.0$ & $2.98+1.28 i$ & $6.17+1.61 i$ & $9.34+1.81 i$ & $12.4+1.96 i$ & $15.6+2.07 i$ \\
(Müller) $D=1.0$ & $2.55+0.80 i$ & $5.60+0.86 i$ & $8.70+1.87 i$ & $11.83+0.87 i$ & $14.96+0.87 i$ \\
(Asymptotic) $D=1.0$ & $2.52+0.70 i$ & $5.56+0.70 i$ & $8.68+0.70 i$ & $11.81+0.70 i$ & $14.94+0.70 i$ \\
(Müller) $D=5.0$ & $2.44+0.42 i$ & $5.53+0.43 i$ & $8.66+0.43 i$ & $11.8+0.43 i$ & $14.9+0.43 i$ \\
(Asymptotic) $D=5.0$ & $2.43+0.40 i$ & $5.53+0.40 i$ & $8.66+0.40 i$ & $11.8+0.40 i$ & $14.9+0.40 i$ \\
(Müller) $D=20$ & $2.41+0.22 i$ & $5.52+0.22 i$ & $8.65+0.22 i$ & $11.8+0.22 i$ & $14.9+0.22 i$ \\
\hline
\end{tabular}

Any incident wave can be expressed in terms of a linear superposition of Dirac functions; the response, $\varphi$, will then be

$\varphi(\lambda, \sigma)=\int_{0}^{t(\lambda, \sigma=1)} G\left(\lambda, t_{0}, \sigma\right) \eta^{I}\left(t_{0}, x=1\right) \mathrm{d} t_{0}$.

Because of the linearisation at the toe of the slope, the upper limit of the integration, $t(\lambda, \sigma=1)$, in Eq. (20) can be simply replaced by $\lambda$. We will use $\widetilde{G}\left(\omega, t_{0}, \sigma\right)$ to recover $G\left(\lambda, t_{0}, \sigma\right)$. Accordingly the potential will become

$$
\begin{aligned}
& \varphi(\lambda, \sigma)= \\
& \int_{0}^{\lambda} \mathrm{d} t_{0} \int_{-\infty}^{\infty} \widetilde{G}\left(\omega, t_{0}, \sigma\right) \eta^{I}\left(t_{0}, x=1\right) \exp (i \omega \lambda) \mathrm{d} \omega,
\end{aligned}
$$

where the integration over the frequencies will be transformed to a series of residues. This is not a closed integral over the complex plane but a real line integral between $-\infty$ and $\infty$, except $\omega=0$ which we circumvent with an infinitesimal semicircle on the lower half-plane. The reason we use the lower half-plane is that we want Green's function to vanish for negative values of $\lambda$. The whole integral can be cast into a closed integral by connecting $\infty$ to $-\infty$ along a semicircle on the upper complex plane and can be calculated using a residue summation. Here, $\omega=0$ is not the only pole. As a matter of fact $\widetilde{G}\left(\omega, t_{0}, \sigma\right)$ has many poles in the upper halfplane (see Eq. 19). These poles are symmetrical with respect to the imaginary axis. Our aim is to understand the excitation of these free modes by the incident waves.

It is important to note that as $x \rightarrow \infty$ these free modes diverge. However, this is not a problem if one wishes to find a solution in the coastal zone. We will expand the reflected wave in terms of these free modes. For the numerical calculation, the free mode expansion is truncated at a finite term $N$. This finite series also diverges for $x \rightarrow \infty$, thus the truncation error between the real reflected wave and the finite series expansion grows as $x$ increases, but this is also not a problem if one wishes to find the wave field near the coast because the discrepancy (or error) propagates towards the ocean.
To perform the free mode expansion approach, let us rewrite the convolution (Eq. 20) as

$$
\begin{aligned}
& \varphi(\lambda(t, x), \sigma(t, x))= \\
& \frac{1}{2 \pi} \int_{0}^{t} \mathrm{~d} t_{0} \int_{-\infty}^{\infty} \frac{2 \sqrt{D+1}}{\omega\left(i \sqrt{D+1} J_{0}(2 \omega)-J_{1}(2 \omega)\right)} \\
& \quad J_{0}(2 \omega \sigma) \eta^{I}\left(t_{0}, x=1\right) \times \exp \left(i \omega\left(t-t_{0}\right)\right) \mathrm{d} \omega .
\end{aligned}
$$

In an effort to calculate solitary wave run-up, Synolakis (1987) evaluated a similar integral in his linear approach. However, his integral was tasked with directly computing the free surface, $\eta$, rather than the potential and consequently did not have a singularity at $\omega=0$. The Fourier transform of the particular solitary wave Synolakis (1987) considered approached zero so fast along the infinite-radius integration contour that he was able to close his contour on the lower half-plane (upper half-plane in his convention). Thus, his complex integration loop did not contain any of the complex frequencies we mentioned above. The only poles that remained within his closed contour were those of the Fourier transform of the solitary wave he considered and were, therefore, independent of the geometry. His technique is limited to this particular incident wave forcing. In his article, Synolakis (1987) expressed the solution in terms of a summation over discrete frequencies, but these frequencies can not be interpreted as free mode frequencies not only because they are independent of the geometry but also because the result is only valid for times smaller than a critical time $t_{\mathrm{c}}$. To see this, let us write down the integral (given as equation 2.6 in Synolakis, 1987) in our convention for $D=0$ :

$$
\eta(t, x)=\frac{1}{2 \pi} \int_{-\infty}^{\infty} \frac{2 \Phi(\omega) J_{0}(2 \omega \sqrt{x})}{\left(i J_{0}(2 \omega)-J_{1}(2 \omega)\right)} \exp (i \omega t) \mathrm{d} \omega,
$$

where $\Phi$ is the Fourier transform of incident wave. In the integrant here, the exponential term $\exp (i \omega t)$ diverges on the lower semicircle. This divergence is counter-balanced by the Fourier transform of the incident wave for $t<t_{\mathrm{c}}$ but not later.

To do our calculations for wave evolution, we will need the non-vanishing poles of $A_{0}(\omega)$ which we calculate using the Müller scheme (see Press et al., 2007). For large $D$ an asymp- 
totic approximation is presented in Appendix A. A comparison between these two approaches is displayed in Table 1 .

As seen in this table, as $D$ increases, radiation towards the ocean becomes less efficient, therefore making the complex parts of the roots smaller. The real parts also decrease as $D$ increases. This is because the waves that propagate from the shore towards the ocean create reflecting waves continuously because of the variable depth of the slope over which they are travelling. However, we have radiation damping in our case and some of the waves that reach the toe of the channel escape from the sloping part of the channel. In the absence of radiation damping, all waves reaching the toe of the channel would reflect back. These waves contribute to standing waves of low frequencies over the sloping channel because of the long distance they travel. Consequently, the relative weight of this low frequency component would increase if there is no or little radiation. In the case of any non-uniform bathymetry, at the high-frequency limit, ray theory can be used and the reflections will become minimal. This explains why the damping factor of the higher modes (imaginary parts of the eigenfrequencies) become larger. In the real geophysical settings where the discontinuities are less abrupt than the geometry depicted here (such as the edges of coastal shelves), the short waves will cross over the toes of the slopes, essentially without reflection. In Model-2 where we consider an opening to a semi-infinite ocean, short waves get reflected even for minor depth discontinuities; hence, the formulation we developed so far is important.

The geometry considered by Stefanakis et al. (2015) features two consecutive slopes. If were to fix the slope angles in their work to a single value, the resulting geometry would be the same as ours. The frequencies that they would have come up with in their solutions would have been the real roots of $J_{0}(2 \omega)$. These frequencies are substantially smaller than the real parts of the frequencies we calculate ( 2.98 versus 2.4 for the fundamental mode) with radiation damping.

Now let us return to the integral (Eq. 22). This integral can be written completely in terms of $\lambda$ and $\sigma$ as

$$
\begin{aligned}
& \varphi(\lambda, \sigma)= \\
& \frac{1}{2 \pi} \int_{0}^{\lambda-2(1-\sigma)} \mathrm{d} t_{0} \int_{-\infty}^{\infty} \frac{2 \sqrt{D+1} J_{0}(2 \omega \sigma)}{\omega\left(i \sqrt{D+1} J_{0}(2 \omega)-J_{1}(2 \omega)\right)} \\
& \eta^{I}\left(t_{0}, \sigma=1\right) \times \exp \left(i \omega\left(\lambda-t_{0}\right)\right) \mathrm{d} \omega
\end{aligned}
$$

for $\lambda>2(1-\sigma)$ because of the causality ( $\varphi$ becomes zero otherwise, because the disturbance takes $2(1-\sigma)$ to travel from the toe of the slope to the point $\sigma$ ). A residue summation transforms Eq. (24) into

$\varphi=\left(2 \int_{0}^{\lambda-2(1-\sigma)} \mathrm{d} t_{0} \eta^{I}\left(t_{0}, x=1\right)\right)$

$$
\begin{aligned}
& +\sum_{k=-\infty, k \neq 0}^{+\infty} \frac{i \sqrt{D+1} J_{0}\left(2 \omega_{k} \sigma\right)}{\omega_{k}\left(i \sqrt{D+1} J_{0}^{\prime}\left(2 \omega_{k}\right)-J_{1}^{\prime}\left(2 \omega_{k}\right)\right)} \\
& \times \quad \int_{0}^{\lambda-2(1-\sigma)} \mathrm{d} t_{0} \eta^{I}\left(t_{0}, x=1\right) \exp \left(i \omega_{k}\left(\lambda-t_{0}\right)\right),
\end{aligned}
$$

where $J_{0}{ }^{\prime}$ and $J_{1}{ }^{\prime}$ are the derivatives of $J_{0}\left(2 \omega_{k}\right)$ and $J_{1}\left(2 \omega_{k}\right)$ with respect to $2 \omega_{k}$. The first integral in Eq. (25) comes from the residue associated with the pole at $\omega=0$. It is calculated by using the fact that $J_{0}(0)=1$ and $J_{1}(0)=0$. Using the Leibniz rule, the partial derivatives of the potential are then

$$
\begin{aligned}
\partial_{\lambda} \varphi= & 2 \eta^{I}(\lambda-2(1-\sigma), x=1) \\
& +\sum_{k=-\infty, k \neq 0}^{\infty} \frac{i \sqrt{D+1} J_{0}(2 \omega \sigma)}{\omega_{k}\left(i \sqrt{D+1} J_{0}^{\prime}\left(2 \omega_{k}\right)-J_{1}^{\prime}\left(2 \omega_{k}\right)\right)} \\
& \times \eta^{I}(\lambda-2(1-\sigma), x=1) \exp \left(2 i \omega_{k}(1-\sigma)\right) \\
& +\sum_{k=-\infty, k \neq 0}^{\infty} \frac{-\sqrt{D+1} J_{0}\left(2 \omega_{k} \sigma\right)}{i \sqrt{D+1} J_{0}^{\prime}\left(2 \omega_{k}\right)-J_{1}^{\prime}\left(\left(2 \omega_{k}\right)\right.} \\
& \quad \int_{0}^{\lambda-2(1-\sigma)} \mathrm{d} t_{0} \eta^{I}\left(t_{0}, x=1\right) \exp \left(i \omega_{k}\left(\lambda-t_{0}\right)\right)
\end{aligned}
$$

and

$$
\begin{aligned}
\partial_{\sigma} \varphi= & 4 \eta^{I}(\lambda-2(1-\sigma), x=1) \\
& +\sum_{k=-\infty, k \neq 0}^{\infty} \frac{2 i \sqrt{D+1} J_{0}\left(2 \omega_{k} \sigma\right)}{\omega_{k}\left(i \sqrt{D+1} J_{0}{ }^{\prime}\left(2 \omega_{k}\right)-J_{1}{ }^{\prime}\left(2 \omega_{k}\right)\right)} \\
& \times \exp \left(i \omega_{k} 2(1-\sigma)\right) \eta^{I}(\lambda-2(1-\sigma), x=1) \\
& +\sum_{k=-\infty, k \neq 0}^{\infty} \frac{-2 i \sqrt{D+1} J_{1}\left(2 \omega_{k} \sigma\right)}{i \sqrt{D+1} J_{0}{ }^{\prime}\left(2 \omega_{k}\right)-J_{1}{ }^{\prime}\left(2 \omega_{k}\right)} \\
\lambda-2(1-\sigma) & \int_{0}^{1-\operatorname{din} t_{0} \exp \left(i \omega_{k}\left(\lambda-t_{0}\right)\right) \eta^{I}\left(t_{0}, x=1\right) .}
\end{aligned}
$$

The equation given in Eq. (9) is, in essence, the linear wave equation with cylindrical symmetry. Because of this, the partial derivative of its regular solution with respect to $\sigma$ must be zero at $\sigma=0$. A quick inspection of Eq. (27) reveals that the terms in the first and second line are not equal to zero individually, and their collective sum will involve a truncation error. This truncation error is subject to an amplification in the estimation of $\partial_{\sigma}(\varphi) / \sigma$ which is used to calculate both $\eta$ and $u$. To remedy this, we use the fact that, near $\sigma=0$, the value of $\partial_{\sigma} \varphi / \sigma$ is approximately equal to $2 \partial_{\lambda \lambda}^{2} \varphi$ due to L'Hôspital's rule. Hence, we use the numerical derivative of Eq. (26) to find the nonlinear contribution to run-up.

\section{Resonance sensitivity for Model-1}

In this section we consider a monochromatic incident wave of type $\eta_{0}^{I}(\omega) \sin (\omega(t+(x-1) / \sqrt{D+1}))$. We will study the 
evolution in the large time limit, namely the standing wave regime. The transient regime will be discussed in the next subsection. The resulting wave on the slope in the linear approximation will then be

$$
\begin{aligned}
\eta(t, x)= & \left(A_{0}(\omega) \frac{\exp (i \omega t)}{2}\right. \\
& \left.+A_{0}(-\omega) \frac{\exp (-i \omega t)}{2}\right) \omega J_{0}(2 \omega \sqrt{x})
\end{aligned}
$$

for $t \rightarrow \infty$. This equation follows naturally from Eq. (15), where, instead of evaluating the integral, we add the two contributions coming from $\omega$ and $-\omega$. Taking into account that $J_{0}(0)=1$, the associated run-up, $r=\eta(t, \sigma=0)$, becomes

$\omega\left|A_{0}(\omega)\right| \cos \left(\omega t+\phi_{A}\right)$

in the linearised theory. Here, $\phi_{A}$ is the argument of complex number $A_{0}(\omega)$. In a real situation that would occur in nature where the monochromatic incident wave gets generated at a particular instant $t=0$, the expression

$\omega\left|A_{0}(\omega)\right|$

in Eq. (29) provides a limiting value of amplitude of oscillation of the run-up. This limiting amplitude, normalised to the amplitude of the incident wave, is displayed in Fig. 2 as a function of $2 \omega$, where $\omega$ is the frequency of the incident wave. In this figure local maxima of the limiting amplitude of the run-up can be observed for $D=1$ and $D=5$ but not for $D=0$, where this amplitude steadily increases with the frequency of the incident wave. Therefore, there is no resonance for $D=0$.

In the limit of large $D$, the local maxima of the limiting amplitude of the run-up occur at the frequencies where $J_{0}(2 \omega)=0$ and the value of the limiting amplitude of the oscillation of the run-up at those maxima is given as

$2 \sqrt{D+1}\left|\eta_{0}^{I}\left(2 \omega_{k}\right) / J_{1}\left(2 \omega_{k}\right)\right|$,

where $2 \omega_{k}$ is the $k$ th root of the Bessel function $J_{0}$. Hence, the run-up sensitivity to $\omega$ increases as $D$ increases.

One last remark relates to the power laws for run-up, provided by Didenkulova et al. (2009). The Fig. 2 shows that, when $D$ is large, the denominator of Eq. (16) has roots that are closer to the real axis. This essentially means that for large $D$ explicit relations for power laws might not be possible to derive.

\subsection{Transient regime}

The resonant phenomena we discussed above do not set in immediately upon the entrance of the incident wave into the slope region. It is important to know how fast the limiting amplitude of oscillation of the run-up will be reached, because in a real situation the incident wave will have a finite duration, a fact that was not taken into account in the large time limit analysis.

For that purpose the residue series in Eq. (26) must be evaluated. Here, the incident wave is taken as $\eta_{0}^{I} \sin (\omega(t+(x-$ 1) $/ \sqrt{D+1}) \theta(t-(x-1) / \sqrt{D+1})$, where $\theta$ is the Heaviside step function. The resulting run-up is displayed in Fig. 3. A good agreement between run-up obtained from residues summation and the fast Fourier transform approach can be seen in this figure (continuous and dashed curves respectively). The limiting amplitude for the run-up is almost reached after just one oscillation (see the horizontal limiting line on the top part of the figure). In Fig. 4 the run-up is displayed as a function of time for incident waves of three different frequencies. In this figure no significant change in run-up can be observed as the frequency of the incident wave deviates from the resonant frequency. This is due to the fact that the depth discontinuity $D$ is zero. On the other hand in the bottom part of Fig. 4, where $D$ is equal to 5, the run-up increases as the incident wave frequency approaches the resonant frequency. A comparison between the upper and bottom parts of Fig. 4 reveals that in the bottom figure it takes longer for the run-up to reach its limiting value. This is due to the fact that for larger $D$ the imaginary part of the frequency of the free mode is smaller. This means that if the incident wave excitation were to be cut at a given time, the standing wave oscillations over the slope would last longer (before eventually decaying due to the radiation) when $D$ is larger. Similarly, for large $D$, it also takes longer for the standing wave regime to reach its limiting amplitude. It is also important to note that in both parts of Fig. 4 the initial time derivative of the run-up is very large, leading to the glitch at $t=6$ for the $D=5$ case on the bottom. No such glitch exists $D=0$ because there is minimal reflection from the toe. In nature, waves do not "switch on" at once at a given time. Therefore, their initial profiles do not have discontinuous spatial derivatives. As a matter of fact, such discontinuities will trigger short-frequency waves which can not be properly modelled using shallow water approach anyway. The discontinuity mentioned here is seen much more clearly in the shoreline velocity field in Fig. 5.

\subsection{Nonlinear effects}

We assume the incident wave to be linear. Considering that we are dealing with a monochromatic incident wave, this makes sense, because any nonlinearity over the flat part of the ocean would have generated higher harmonics during the propagation. As long as the waves do not break, the nonlinearity arising from the shoaling over the slope is accounted for by the CG approach. This particular nonlinearity, as indicated by Pelinovsky and Mazova (1992), does not affect the maximum shoreline velocity; it does, on the other hand, affect the timing of the maximum. After the CG transformation the equations become linear the only remaining nonlinearity in the $(\lambda, \sigma)$ space for the run-up is the $u_{\mathrm{s}}^{2} / 2$ term in Eq. (7), 


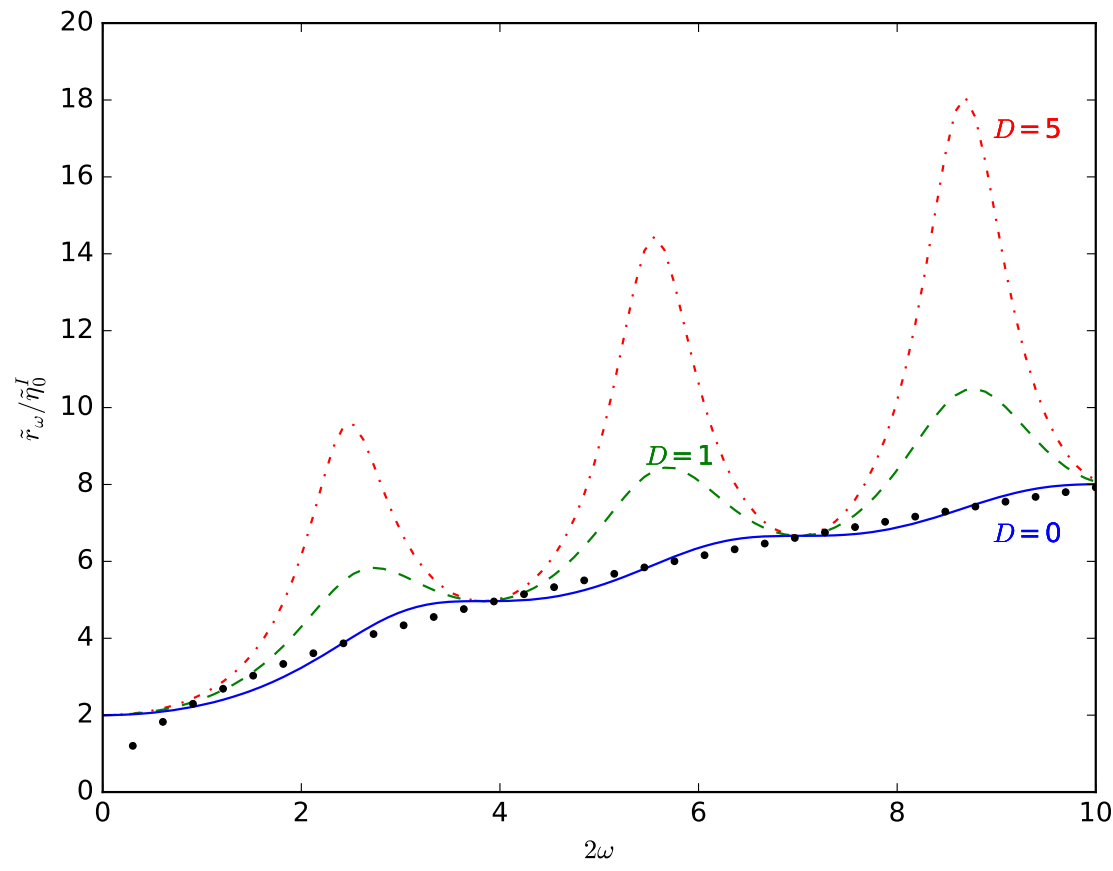

Figure 2. The limiting amplitude of run-up, $\widetilde{r}_{\omega}$, normalised to the amplitude of the incident wave is shown as a function of the frequency of the incident wave multiplied by 2 for Model-1. The depth discontinuity $D$ is 0 for the blue continuous curve, 1 for the green dashed curve and 5 for the red dotted-dashed curve. The black bullets correspond to the limiting run-up given in the expression Eq. (37), normalized to $\tilde{\eta}_{0}^{I}$, for the infinite slope. Here, $\sigma_{0}$ is taken to be one.

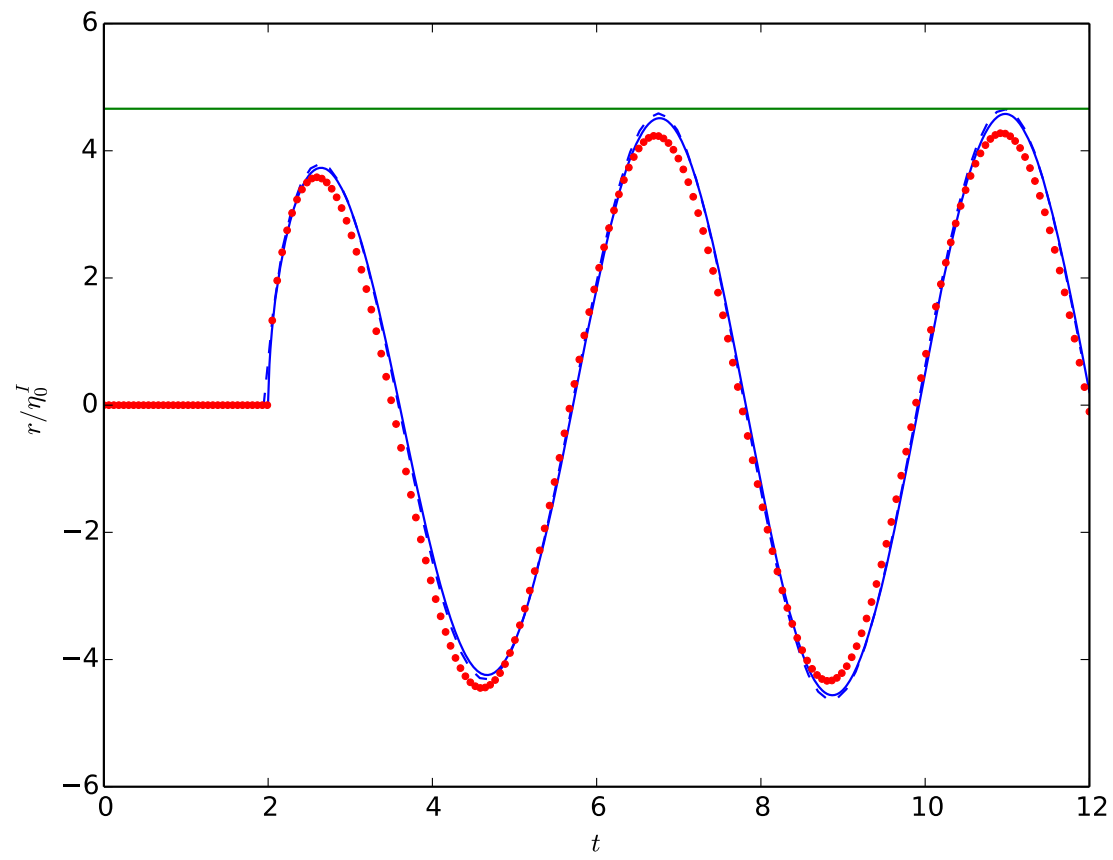

Figure 3. Continuous and dashed blue curves display the run-up normalised to the amplitude of incident wave for Model- 1 with $D=0$. The frequency of the incident wave is equal to the real part of the first-mode resonant frequency $\omega_{1}$ (see Table 1). The continuous blue curve is obtained from the series of residues (see Eq. 26). The dashed blue curve is the run-up calculated using the fast Fourier transform approach. The green horizontal line on the top part of the figure is the limiting amplitude given by $\omega^{I}\left|A_{0}\left(\omega^{I}\right)\right| / \eta_{0}^{I}$, where $\omega^{I}$ is the frequency of the incident wave. The red bullets are the run-up produced by a wave maker on an infinite constant slope. The action of the wave maker is represented by the "tsunamigenic" seafloor motion given by $h(t, x)=-2 \eta_{0}^{I} \delta(x-1) \cos \left(\Re \omega_{1} t\right) \theta(t) / \Re \omega_{1}$, where $\theta$ is the Heaviside function, $h$ is the seafloor uplift and $\Re$ denotes the real part. 

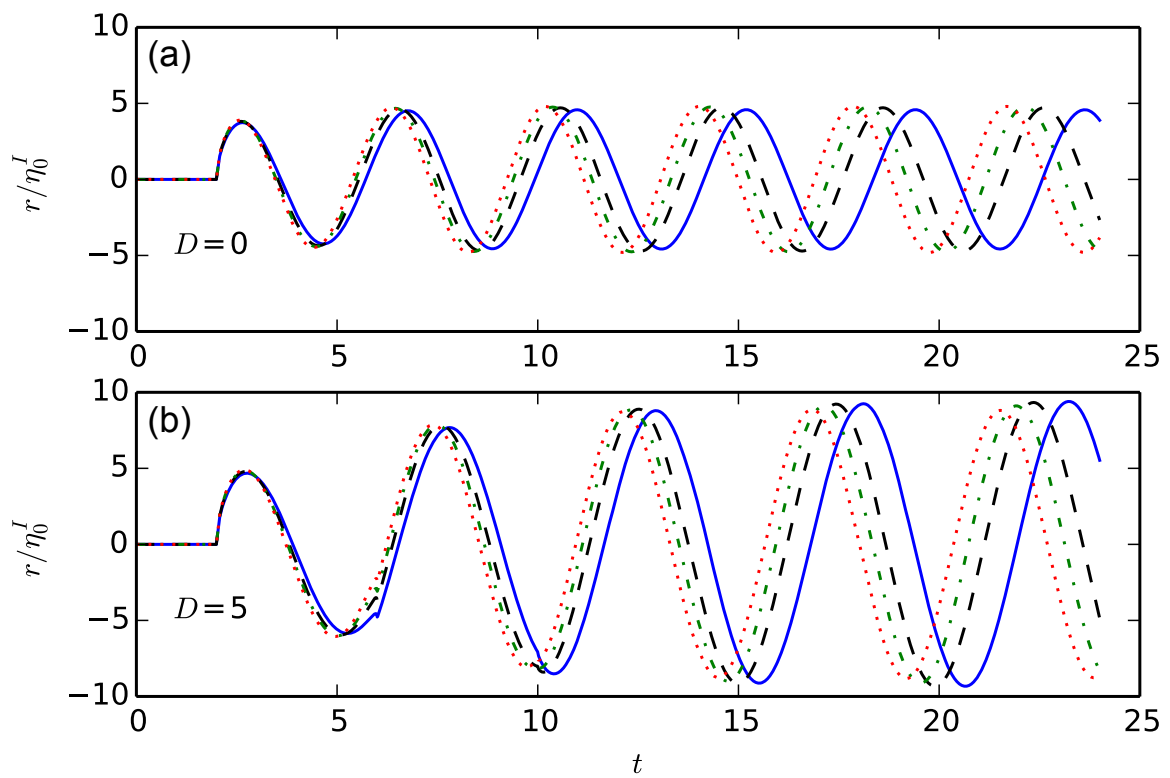

Figure 4. The run-up normalised to the amplitude of the incident wave for Model-1. In panel (a), the frequency of the incident wave is $\Re \omega_{1}(D=0)$ for the blue continuous curve, $1.05 \Re \omega_{1}(D=0)$ for black dashed curve, $1.075 \Re \omega_{1}(D=0)$ for the green dashed-dotted curve and $1.1 \Re \omega_{1}(D=0)$ for the red dotted curve. In panel $(\mathbf{b})$ the frequencies $\Re \omega_{1}(D=0), 1.05 \Re \omega_{1}(D=0), 1.075 \Re \omega_{1}(D=0)$ and $1.1 \Re \omega_{1}(D=0)$ have been replaced by $\Re \omega_{1}(D=5), 1.05 \Re \omega_{1}(D=5), 1.075 \Re \omega_{1}(D=5)$ and $1.1 \Re \omega_{1}(D=5)$ respectively. Depth discontinuity $D$ is 0 in panel (a) and 5 in panel (b). Refer to Table 1 for the values of $\omega_{1}$. All curves are obtained using series of residues. Note that in the bottom figure the wave arrives at the shore at $t=2$ and the first reflection from the toe of the slope reaches the shore at $t=6$, for which the continuous plot includes a slight glitch. In the main text we elaborate on this glitch.
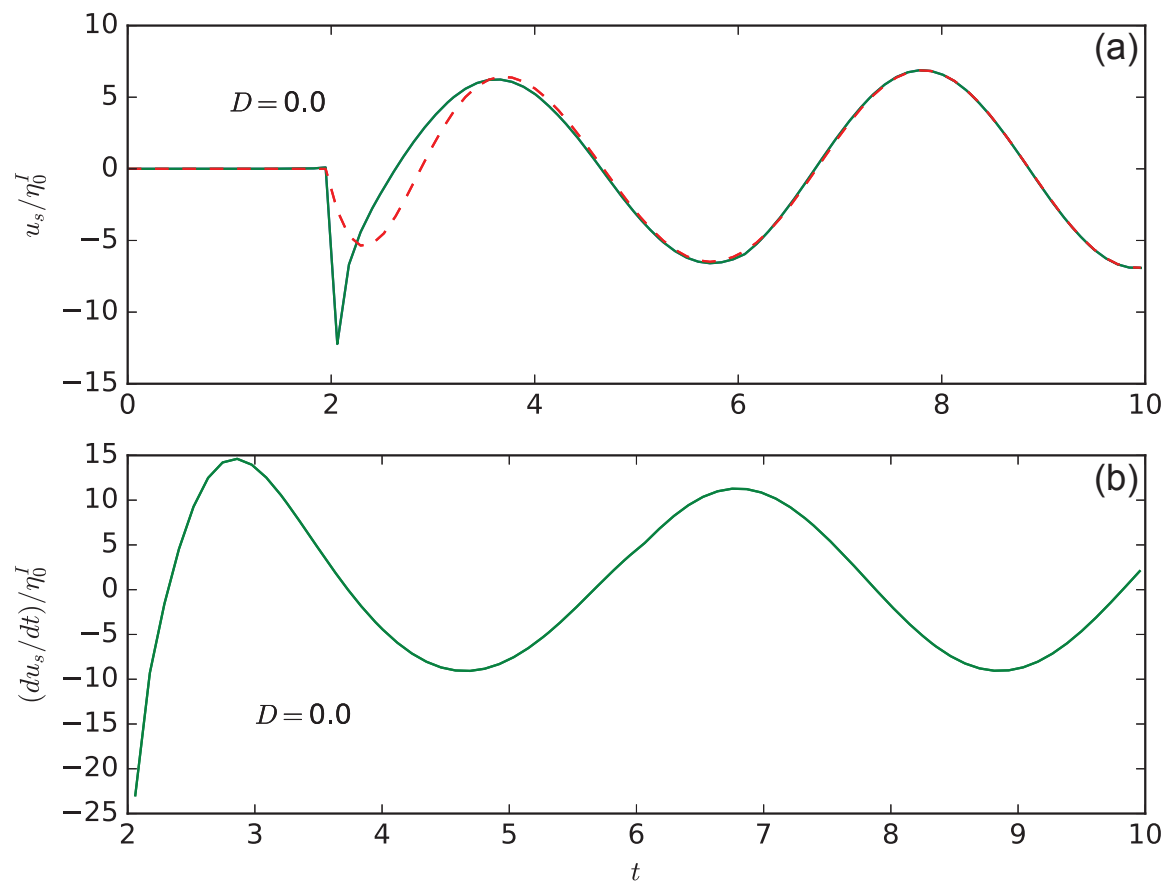

Figure 5. Panel (a) is the shoreline velocity $u_{\mathrm{S}}$ normalised to the amplitude of the incident wave as a function of $t$ (Model 1). The frequency of the incident wave is $\Re \omega_{1}$. The continuous green curve is the shoreline velocity corresponding to incident wave $\eta=\eta_{0}^{I} \sin \left(\Re \omega_{1}(t+(x-\right.$ 1) $/ \sqrt{D+1}) \theta(t+(x-1) / \sqrt{D+1})$. The red dashed curve is the same for the incident wave that has been smoothed by multiplying it by $\tanh (t+(x-1) / \sqrt{D+1})$. Panel (b) is the time derivative of the shoreline velocity for the smoothed incident wave. 


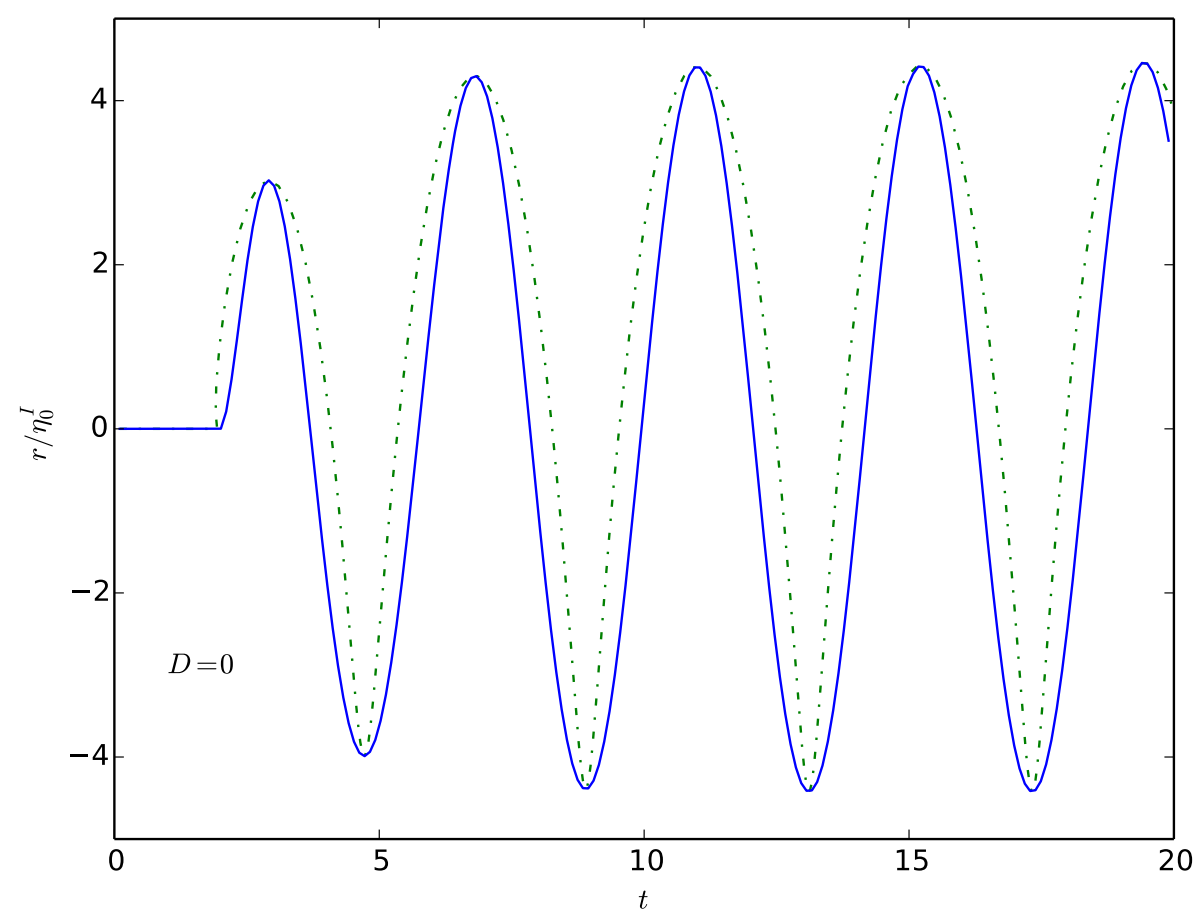

Figure 6. Run-ups normalized to the amplitude of the incident wave for Model-1. The incident wave is in the form $\eta=\eta_{0}^{I} \sin (\omega(t+(x-$ $1) / \sqrt{D+1}) \theta(t+(x-1) / \sqrt{D+1}) \tanh (t+(x-1) / \sqrt{D+1})$. The frequency of the incident wave is $\Re \omega_{1}(D=0)$. The blue continuous curve is the linear run-up. The dashed-dotted green curve includes nonlinear effects associated with $\mathrm{CG}$ transform $\left(r=\partial_{\lambda} \varphi-0.5 u_{\mathrm{S}}^{2}\right.$ and $t=\lambda+u_{\mathrm{S}}$ ). The amplitude of the incident wave is $1 / 15$. Nonlinear effects due to the boundary condition at the toe of the slope have been neglected.

with $u_{\mathrm{s}}$ being the fluid velocity on the shoreline. Figure 6 shows the difference between the two values of run-up calculated by taking and not taking this $u_{\mathrm{s}}^{2} / 2$ term into account. Another subtlety in this figure is that for the nonlinear case the time is given as $t=\lambda+u_{\mathrm{s}}$.

For $t \rightarrow \infty$, the nonlinear run-up is given as $\omega\left|A_{0}\right| \sin (\omega \lambda)-0.5 u_{\mathrm{s}}^{2}$, which approximately becomes

$$
\begin{array}{r}
\omega\left|A_{0}\right|\left(\sin (\omega t)-\omega u_{\mathrm{s}} \cos (\omega t)\right)-\frac{1}{2} u_{\mathrm{s}}^{2}= \\
\left|A_{0}\right| \omega \sin (\omega t)+\omega^{4}\left|A_{0}\right|^{2} \frac{\cos (2 \omega t)+1}{4} .
\end{array}
$$

The reason we performed this expansion with respect to small shoreline velocity is that we eventually want to compare the nonlinear effects on the run-up, caused by the nonlinear boundary condition at $x=1$ with the contribution by the nonlinear terms in the above equation.

At the near-resonant frequencies nonlinear effects will become important even in the deep part of the slope, rendering the linearised boundary conditions that we apply at $x=1$ invalid, compromising the relations for $A_{0}(\omega)$ and $R(\omega)$ (Eqs. 16 and 17). In Appendix B we give the results from a first-order perturbative approach to correct the boundary conditions for nonlinearity. We performed a comparison between the nonlinearity brought in by the boundary condition and that caused by the nonlinear part of Eq. (32). The details of this perturbative analysis can be found in Appendix B. The results suggest that the first-order correction associated with the nonlinearity of the boundary condition at $x=1$ is given by

$$
\eta^{(1)}=\left\{\begin{array}{l}
N^{(1)} \cos (2 \omega t) J_{0}(4 \omega \sqrt{x})+C^{(1)} \\
\quad \text { for } x<1 \\
R^{(1)} \cos \left(2 \omega t-\frac{(x-1)}{\sqrt{D+1}}\right)+\Theta^{(1)} \theta\left(t-\frac{x-1}{\sqrt{D+1}}\right), \\
\quad \text { for } x>1 .
\end{array}\right.
$$

where the superscripts refer to the first-order perturbation. When $D$ becomes very large, at the near-resonant frequencies $\left(J_{0}(2 \omega) \approx 0\right)$, the time-dependent and time-independent parts of the solution for $\eta$ over the slope respectively become

$$
N^{(1)} \approx \frac{1}{4}\left|A_{0}\right|^{2} \omega^{2} \frac{J_{1}^{2}(2 \omega)}{J_{0}(4 \omega)}
$$

and

$C^{(1)}=\frac{\left|A_{0}\right|^{2} \omega^{2}}{4} J_{1}^{2}(2 \omega)$.

These indicate that the nonlinearity brought in by the nonlinear part of Eq. (32) is comparable to that caused by the 
boundary conditions at $x=1$ only for the first mode and when $D$ is infinite. This is to be expected, because shoaling is insignificant for the first mode. The wave must travel a distance of several wavelengths in order to experience significant amplification associated with shoaling. For the first mode $(2 \omega \approx 2.4)$ the analysis yields $N^{(1)} \approx-0.41\left|A_{0}\right|^{2}$ and $C^{(1)} \approx 0.097$. The fact that $N^{(1)}$ is a negative number, taking into account that the $\cos (2 \omega t)$ term in Eq. (33) for $x<1$ takes the value -1 for maximum run-up (because with the phase convention adopted in this section the run-up is proportional to $\sin (\omega t)$ in linear approximation), means that the nonlinearity at seaward boundary condition increases the run-up slightly (see Antuono and Brocchini, 2007 for a similar conclusion reached using a different mathematical approach). The coefficient of the second harmonic in Eq. (32) is $1.4\left|A_{0}\right|^{2}$. For the higher modes the dominance of the Eq. (32) nonlinear effect associated with shoaling increases rapidly because of the fourth power of $\omega$.

\section{Resonance for infinite slope}

In this section we investigate the resonant frequencies of the waves produced by a wave maker placed on an infinite, constant slope. The reason for this practice is that the work that claims significant resonance (Stefanakis et al., 2011) considered an infinite slope. In the analysis we will allow the waves to progress in the offshore direction, unrestricted. When the wavelength produced by the wave maker matches the distance of the wave maker to the shoreline, one might expect a resonance to occur. To see if this is really the case, let us go into a little further detail about the nature of the wave maker.

When the water is sufficiently deep, in the vicinity of the wave maker, linear shallow water equations will apply. The effect of the wave maker, which starts its action at a given time, will be equivalent to hypothetical volume injections and suctions at a rate $2 \eta_{0}^{I} \sqrt{\text { depth }} \sin (\omega t)=\left(2 \eta_{0}^{I} \sigma_{0}\right) \sin (\omega t)$, where $\sigma_{0}^{2}$ is the horizontal distance between the wave maker and the shoreline. Consequently, two waves of equal amplitude of $\eta_{0}^{I}$ start moving in opposite directions if $\omega$ is not smaller than $\sigma_{0}^{2}$. For $\omega \rightarrow 0$ the asymmetry introduced by the slope becomes significant, and the wave maker radiates essentially in the offshore direction (see the black bullets Fig. 2). Due to the superposition principle, the action of the wave maker will not prevent the waves reflected from the shore from freely propagating in the offshore direction. The free surface response to such a source on the slope is given in Eq. (2.13) in Özeren and Postacioglu (2012). To adapt the source in Özeren and Postacioglu (2012) to our case, the expression $S(\widetilde{\sigma}, \widetilde{\lambda})$ needs to be replaced by $2 \eta_{0}^{I} \sin (\omega \widetilde{\lambda}) \delta\left(\widetilde{\sigma}-\sigma_{0}\right) \sigma_{0}$, where $\widetilde{\sigma}$ and $\widetilde{\lambda}$ are integration variables. In Fig. 3, the red bullets show the run-up associated by such a source over an infinite slope as a function of time. For $t \rightarrow \infty$ a simple analytical solution that satisfies the radiation condition (wave progressing in $+x$ direction for $x \gg x_{0}$ ) can be found for a source of the form mentioned above. The presence of the source at $x_{0}$ induces a discontinuity in the fluid velocities such that

$\sigma_{0}\left(u\left(t, x_{0}^{+}\right)-u\left(t, x_{0}^{-}\right)\right)=2 \eta_{0}^{I}(\omega) \sin (\omega t)$

and

$\eta\left(t, x_{0}^{+}\right)=\eta\left(t, x_{0}^{-}\right)$,

where $x_{0}$ is both the horizontal position of the source and the non-dimensional depth at that position. The corresponding solution as $t \rightarrow \infty$ is then given as

$\eta=\left\{\begin{array}{c}-2 \omega \pi \sigma_{0} \eta_{0}^{I}\left(J_{0}(2 \omega \sigma)\left(Y_{0}\left(2 \omega \sigma_{0}\right) \cos (\omega \lambda)\right.\right. \\ \left.\left.-J_{0}\left(2 \omega \sigma_{0}\right) \sin (\omega \lambda)\right)\right) \text { for } \sigma<\sigma_{0} \\ -2 \omega \pi \sigma_{0} \eta_{0}^{I} J_{0}\left(2 \omega \sigma_{0}\right)\left(Y_{0}(2 \omega \sigma) \cos (\omega \lambda)\right. \\ \left.-J_{0}(2 \omega \sigma) \sin (\omega \lambda)\right) \text { for } \sigma>\sigma_{0},\end{array}\right.$

where $Y_{0}$ is the Bessel function of the second kind. For $\sigma \gg \sigma_{0}$ the relation given in Eq. (36) is proportional to $\sin (\omega(t-2 \sqrt{x})) / \sqrt{x}$. The amplitude of the resulting run-up then becomes

$2 \omega \pi \sigma_{0} \eta_{0}^{I} \sqrt{Y_{0}^{2}\left(2 \omega \sigma_{0}\right)+J_{0}^{2}\left(2 \omega \sigma_{0}\right)}$,

and for large frequencies it is reduced to $2 \sqrt{\pi \omega \sigma_{0}} \eta_{0}^{I}$. The black-dotted curve in Fig. 2 shows this expression normalized by $\eta_{0}^{I}$ as a function of $2 \omega$ for $\sigma_{0}=1$. As seen in this figure, the resonance for the infinite slope is almost nonexistent. It is also worthwhile to mention that the run-up tends to zero for $\omega \rightarrow 0$ for the infinite slope. The reason for this is that the wave generated during one period of the forcing activity spreads over to an infinite distance offshore. It is clear from Fig. 2 that the wave generated by the artificial source/sink that we introduce satisfactorily emulates an incident wave if the wavelength of the generated wave is less than the distance to the shore.

\section{Normally incident wave from a 2-D ocean into a bay}

Analytical studies that consider run-up in 2-D are rare. Among the most relevant, we can mention Brocchini and Peregrine (1996), who considered oblique nonlinear incident waves over slopes with infinite width, and Brocchini (1998), who investigated the run-up of weakly 2-D solitary pulses. There are also some studies that combine 1-D analytical approaches with 2-D numerical simulations such as Choi et al. (2011). In this section we shall study Model-2. We consider an incident wave of the form $\eta_{0}^{I}(\omega) \exp (i \omega(t+$ $(x-1) / \sqrt{D+1})$ coming from the open ocean into a sloping bay of width $2 a$. This wave will be reflected back by the shallower part of the bay. This reflected wave will then be subject to geometrical spreading in the open ocean. Consequently, the one-dimensional nature of the waves will be lost in the deeper part of the bay. We will now derive a simple mathematical formulation to model these phenomena. 
The governing equations, linearised in the deeper part of the channel and the open sea, for the potential, $\varphi$, read

$$
\omega^{2} \varphi(x, y)=\left\{\begin{array}{l}
\left(\partial_{\sigma \sigma}^{2}+\frac{1}{\sigma} \partial_{\sigma}\right) \varphi(\sigma) \\
\text { for } x \ll 1 \\
\partial_{x}\left(h(x) \partial_{x} \varphi\right)+h(x) \partial_{y y}^{2} \varphi \\
\text { for } 0 \ll x<1 \text { and } \\
\partial_{y} \varphi(x, y)=0 \text { for } y= \pm a \\
(D+1)\left(\partial_{x x}^{2}+\partial_{y y}^{2}\right) \varphi \\
\text { for } x>1 \text { and } \\
\partial_{x} \varphi\left(x=1^{+}, y\right)=0 \text { for }|y|>a
\end{array}\right.
$$

where $h(x)=x$ is the undisturbed depth in our case. For a constant slope beach Mei et al. (2004) page 155 and Zhang and Wu (1999) used the confluent hypergeometric function $M$. We will now adapt their solution to a sloping bay. Let us start by defining a potential inside the channel as

$$
\begin{aligned}
& \varphi_{\omega}(t, x)=A_{0}(\omega) J_{0}(2 \omega \sigma) \exp (i \omega \lambda) \\
& +\sum_{n=1}^{\infty} A_{n}(\omega) \exp \left(-\frac{\pi n x}{a}\right) M\left(-\frac{\omega^{2} a}{2 n \pi}+\frac{1}{2}, 1,2 \pi n x / a\right) \\
& \quad \cos \left(n \pi \frac{y+a}{a}\right) \exp (i \omega t),
\end{aligned}
$$

where the unknown coefficients $A_{0}, A_{1}, \ldots$ are to be determined from the boundary conditions at the mouth of the channel. Note that the solution given in Eq. (39) satisfy the linear shallow water equations in the deeper part of the channel. For $1-x \gg a$ the $y$-dependent part of the solution quickly decays. The cosine term with integer values of $n$ is both symmetrical about the $x$ axis and its derivative with respect to $y$ for $y= \pm a$ is zero, satisfying, therefore, the noflux condition on the channel sides. In the deeper part of the channel where $1-x$ is of the order of $a$, the velocities in $x$ and $y$ directions can be derived from the potential given in Eq. (39) using $u=-\partial_{x} \varphi$ and $v=-\partial_{y} \varphi$ respectively.

The bulk of the incident wave will be reflected back by the solid boundary at $x=1,|y|>a$. Consequently the wave in the open ocean will be $\eta=2 \widetilde{\eta}_{0}^{I} \cos (\omega(t-(x-1) / \sqrt{D+1}))$, perturbed by the waves radiating from the mouth of the channel and the scattering from the channel mouth corners. The potential in the open sea then reads

$$
\begin{aligned}
& \varphi_{\omega}(t, x>1, y)=2 \frac{\widetilde{\eta}_{0}^{I}(\omega) \cos (\omega(x-1) / \sqrt{D+1})}{i \omega} \exp (i \omega t) \\
& +\int_{-a}^{a} \mathrm{~d} \widetilde{y} \frac{S(\omega, \widetilde{y})}{-2 i(D+1)} H_{0}^{(2)}\left(\frac{\omega}{\sqrt{D+1}} \mid(x-1) \hat{\boldsymbol{i}}\right. \\
& \quad+(y-\widetilde{y}) \hat{\boldsymbol{j}} \mid) \exp (i \omega t),
\end{aligned}
$$

where $\hat{\boldsymbol{i}}$ and $\hat{\boldsymbol{j}}$ are unit vectors in the $x$ and $y$ directions respectively. Note that the Hankel function $H_{0}^{(2)}$ satisfies both the linearised wave equation in two dimensions and the radiation condition for $\omega>0$. For $\omega<0$, on the other hand, the Hankel function will be replaced by its complex conjugate alongside the rest of the terms. The integral in Eq. (40) represents the potential of the waves radiating from the mouth of the channel. Function $S(\omega, \tilde{y})$ is the unknown virtual sources distribution along the mouth of the channel. This source distribution will be determined by matching the potentials given in Eqs. (39) and (40).

We want the solution in the open sea to satisfy the no-flux condition at $(x=1,|y|>a)$. According to Mei et al. (2004, p. 194), the solution given in Eq. (40) satisfies this condition as

$(D+1) \frac{\partial}{\partial x} \varphi_{\omega}\left(t, x=1^{+}, y\right)= \begin{cases}0 & \text { for }|y|>a \\ S(\omega, y) & \text { for }|y|<a .\end{cases}$

To match the depth-integrated $u$ along the mouth we need to satisfy

$\left.(D+1) \frac{\partial \varphi_{\omega}(x, y)}{\partial x}\right|_{x=1^{+}}=S(\omega, y)=\left.\frac{\partial \varphi_{\omega}(x, y)}{\partial x}\right|_{x=1^{-}}$.

The second condition to be satisfied is the continuity of $\eta$ itself, across the mouth. This condition reads

$\left.\varphi_{\omega}(x, y)\right|_{x=1^{+}}=\left.\varphi_{\omega}(x, y)\right|_{x=1^{-}}$.

Here, it is important to note that the condition (Eq. 43) is an integral equation for the source distribution. The condition (Eq. 42), on the other hand, is not an integral equation, because the field inside the channel is governed using summations rather than an integral. In order to solve a system that simultaneously involves an integral equation and a set of algebraic equations, we shall expand the source distribution, $S(\omega, y)$, in terms of even-order Legendre polynomials as

$S(\omega, y)=\sum_{n=0}^{N-1} \widetilde{S}_{n}(\omega) P_{2 n}\left(\frac{y}{a}\right)$,

where the even indices of the Legendre polynomial were used to make sure that the solution is symmetric with respect to the symmetry axis of the sloping channel $(y=0)$. The coefficients $\widetilde{S}_{n}(\omega)$ in Eq. (44) and $A_{0}, A_{1}, \ldots, A_{N-1}$ in Eq. (39) (we truncate the series in Eq. 39 to $N-1$ ) are solved in such a way as to minimise the following penalty integral along the mouth:

$$
\begin{aligned}
& \int_{-a}^{a}\left(\left|(D+1) \frac{\partial \varphi_{\omega}\left(x=1^{-}, y\right)}{\partial x}-S(\omega, y)\right|^{2}\right. \\
& \left.+\left|\varphi_{\omega}\left(x=1^{-}, y\right)-\varphi_{\omega}\left(x=1^{+}, y\right)\right|^{2}\right) \mathrm{d} y .
\end{aligned}
$$

Thus, we have $2 N$ unknowns. The integral (Eq. 45) is evaluated numerically using Gauss quadrature. For precision, the number of quadrature points we use is larger than $2 N$. 
The Eqs. (42) and (43) have to be satisfied at all quadrature points, making the resulting system over-determined. We use the weighted least-squares approach to solve this overdetermined system. Algebraically speaking, this simply corresponds to writing down Eqs. (42) and (43) for each quadrature point, multiplying each equation by the square root of the corresponding quadrature weight and solving the resulting linear, over-determined system by using the conventional least-squares method.

Note that in Eqs. (39) and (44), the only terms that are responsible for the net flux from the channel to the open sea are $A_{0}$ and $\widetilde{S}_{0}$, since the following integrals vanish for $n \neq 0$ :

$$
\begin{aligned}
& \int_{-a}^{a} \cos \left(n \pi \frac{y+a}{a}\right) \mathrm{d} y, \\
& \int_{-a}^{a} P_{2 n}(y / a) \mathrm{d} y .
\end{aligned}
$$

The conservation of the net flux requires that $-2 a \omega A_{0} J_{1}(2 \omega)=2 a \widetilde{S}_{0}$, where $J_{1}$ is the Bessel function of order 1 . When the frequency of the incident wave is equal to that of the free oscillation of the system, the coefficients $A_{0}$ and $\widetilde{S}_{0}$ should diverge. In order to determine the frequencies of these free mode oscillations (natural frequencies), the roots of $1 / A_{0}$ are sought in the complex plane using the Müller method.

A quick look at Table 2 reveals that for any mode, with the decreasing channel width $(2 a)$, imaginary parts of the normal mode frequencies decrease. However, for lower modes this effect is slightly more pronounced. This is because the time necessary for the waves to travel over the open ocean across a distance of half-channel width $a$ is equal to $a / \sqrt{D+1}$, and, when this time is much less than the period of a longitudinal oscillation within the sloping channel, the geometrical spreading in the open sea is very efficient. This makes the free surface in the vicinity of the channel mouth almost flat due to the fast escape of the waves, rendering the channel mouth boundary condition effectively a Dirichlet condition with $\eta=0$. Consequently, the waves reaching the mouth reflect very efficiently back towards the shore, limiting the radiation damping.

The rays that the waves follow in the sloping channel are straight lines at the shallower parts, and they bend towards the corners as they get near to the mouth of the channel, due to geometrical spreading (see the streamlines in Fig. C1). If the width of the channel increases, this corner effect will penetrate deeper into the channel, making the rays longer. Longer rays will decrease the frequencies of the free oscillations. This can be observed in Table 2. Overall, because of this ray bending the frequencies in Model-2 are lower than those in Model-1. Having said that, for a fixed value of channel width, if $D$ increases, the discrepancy between Model-1 and Model-2 decreases because for $D \rightarrow \infty$ the free surface in the open ocean side of the domain will be flat for all free modes for both Model-1 and Model-2, making the behaviour the same within the channel.

Now let us turn our attention to the transient response for Model-2. As before, we shall model the transient response to an incident wave of the form $\eta^{I}(t+x / \sqrt{D+1})$, and, as the first step, we shall look at the particular case in which $\eta^{I}$ is a Dirac delta function. For the sloping channel, such a response for the potential $\varphi$ corresponds to the integration of Eq. (39) with respect to $\omega$. This integration reduces down into a residue summation which we shall explain below. Since we are not interested in the $y$-direction dependence of the run-up, we are only interested in $A_{0}$. However, since they are interrelated through the boundary conditions, we end up having to calculate $A_{1}, A_{2}, \ldots A_{n}$ as well, even though we do not need them individually for our physical interpretations.

Remember that in Model-1 we had an analytical expression for $A_{0}$, and therefore also for $\varphi$ (see Eqs. 16 and 14). For Model-2, on the other hand, we do not have a closedform relation for $A_{0}$ except when $\omega \rightarrow 0$. Obviously, when $\omega \rightarrow 0$, the free surface over the slope becomes flat, and in this regime $A_{0}(\omega)$ is approximately equal to $2 \widetilde{\eta}^{I}(\omega) / i \omega$ for both Model-1 and Model-2. Bowers (1977) finds a simple analytical solution for a non-sloping bay that opens into a wider channel, and the wavelength of the incident wave is larger than the width of the larger channel. To approximately adapt his theory for our case, the incident wave frequency must tend to zero. At this limit the solution by Bowers (1977) (see his unnumbered expression for $D$ in page 75 of his article) and our solution both give the run-up as twice the incident wave amplitude. For the rest of the frequencies, $\omega_{k}$, that make $A_{0}$ singular, we simply calculate circular integrals around each $\omega_{k}$ on the complex plane. Consequently, for $\varphi$ we have

$$
\begin{aligned}
\varphi= & \left(2 \int_{0}^{\lambda-2(1-\sigma)} \mathrm{d} t_{0} \eta^{I}\left(t_{0}, x=1\right)\right) \\
& +\sum_{k=-\infty, k \neq 0}^{+\infty} \oint_{c_{k}} A_{0}(\omega) J_{0}(2 \omega \sigma) \mathrm{d} \omega \\
& \times \int_{0}^{\lambda-2(1-\sigma)} \mathrm{d} t_{0} \eta^{I}\left(t_{0}, x=1\right) \exp \left(i \omega_{k}\left(\lambda-t_{0}\right)\right),
\end{aligned}
$$

where $c_{k}$ are integral contours with infinitesimal radii around each $\omega_{k}$. This approach is much faster than calculating the frequency integrals exclusively along the real axis. This is because on the real axis, the integrand becomes oscillatory and the accuracy can only be sustained using small integration steps (in Model-1 we used several tens of thousand integration points to keep the integral stable). This is not feasible because for each integration point, an integral equation (Eq. 45) needs to be solved to calculate the relevant $A_{0}$. On the other 
Table 2. The complex natural frequencies multiplied by 2 are tabulated for Model-2. These are essentially those $2 \omega_{k}$ that are the roots of $1 / A_{0}(\omega)$. In this table those lines with "(int)" list the frequencies calculated using $A_{0}(\omega)$ obtained from minimising the penalty integral (Eq. 45). The lines with "(conf)", on the other hand, use Eq. (48) from the conformal mapping formulation for this quantity. Note that $2 \omega_{k}$ tend to the roots of $J_{0}$ for large $D$ or small $a$.

\begin{tabular}{lrrrrr}
\hline & $2 \omega_{1}$ & $2 \omega_{2}$ & $2 \omega_{3}$ & $2 \omega_{4}$ & $2 \omega_{5}$ \\
\hline$a=0.05, D=0$ (int) & $2.26+0.056 i$ & $5.27+0.126 i$ & $8.32+0.19 i$ & $11.4+0.25 i$ & $14.5+0.31 i$ \\
$a=0.05, D=0$ (conf) & $2.26+0.056 i$ & $5.27+0.126 i$ & $8.32+0.192 i$ & $11.38+0.253 i$ & $14.46+0.310 i$ \\
$a=0.1, D=0$ (int) & $2.18+0.11 i$ & $5.14+0.23 i$ & $8.16+0.34 i$ & $11.2+0.44 i$ & $14.3+0.52 i$ \\
$a=0.1, D=0$ (conf) & $2.18+0.107 i$ & $5.13+0.234 i$ & $8.14+0.346 i$ & $11.17+0.446 i$ & $14.20+0.533 i$ \\
$a=0.1, D=1$ (int) & $2.28+0.057 i$ & $5.30+0.13 i$ & $8.37+0.20 i$ & $11.45+0.26 i$ & $14.6+0.32 i$ \\
$a=0.1, D=5$ (int) & $2.35+0.020 i$ & $5.43+0.045 i$ & $8.53+0.07 i$ & $11.65+0.095 i$ & $14.8+0.12 i$ \\
$a=0.2, D=0$ (int) & $2.05+0.197 i$ & $4.95+0.41 i$ & $7.95+0.58 i$ & $11.0+0.74 i$ & $14.0+0.88 i$ \\
$a=0.2, D=0$ (conf) & $2.05+0.197 i$ & $4.92+0.410 i$ & $7.84+0.572 i$ & $10.75+0.661 i$ & $16.6+0.61 i$ \\
\hline
\end{tabular}

hand, for each $\omega_{k}$, the complex integral $\oint_{c_{k}} A_{0}(\omega) J_{0}(2 \omega \sigma) d \omega$ can be satisfactorily calculated using just four points because the integrand for $\omega \rightarrow \omega_{k}$ becomes proportional to $1 /\left(\omega-\omega_{k}\right)$, which is not oscillatory. A further practicality of this approach for an operational activity such as predicting a storm surge run-up is that these contour integrals are independent of the structure of the incident wave and therefore can be pre-calculated for a particular bay or channel geometry and be injected in during the operational calculations. To relate the run-up to Eq. (47) we follow the same procedure as in Model-1 to compute the partial derivatives of $\varphi$. In Fig. (7) the run-up, shoreline velocity and shoreline acceleration are given as a function of time.

In Fig. C1 the rays not only bend towards the mouth of the channel but they also coalesce. This coalescence reflects certain features that relate to the energy exchange to and from the channel.

To summarise, for both Model-1 and Model-2, when the incident wavelength is larger than the channel length, the run-up amplitude tends to $2 \eta^{I}$; in other words the wave becomes blind to the bathymetric variation. However, an interesting case arises in Model-2 when the wavelength is much larger than the width of the channel but is still less than the length of the channel. In this case an analytical solution can be found using a conformal mapping approach (Mei et al., 2004, p. 218, for a flat-bottomed channel case). In Appendix $\mathrm{C}$ we give the mathematical details of the analysis in which we generalize the conformal mapping approach for the sloping channel with $D=0$. As seen in the Appendix, the corresponding $A_{0}(\omega)$ becomes

$$
\begin{aligned}
A_{0}(\omega)= & \frac{2 \widetilde{\eta}^{I}(\omega)}{i \omega}\left[J_{0}(2 \omega)\right. \\
& \left.+a \omega J_{1}(2 \omega)\left(\frac{-2}{\pi} \ln \left(\frac{e \pi}{2 \Gamma \omega a}\right)+i\right)\right]^{-1},
\end{aligned}
$$

where $\Gamma \approx 1.781$ is the exponential of the Euler-Mascheroni constant. The run-up amplitude is simply then given as
$\omega\left|A_{0}(\omega)\right|$. Figure 8 shows this value as a function of twice the frequency of the incident wave, calculated using both the integral equation and conformal mapping approaches. Kajiura (1977) also used conformal mapping to reach the same conclusion (note that his equivalent figure includes the amplification factor rather than the run-up, and therefore his values are half of those reported here). In this work we essentially extended the solution of Kajiura (1977) to short waves using integral equations.

\section{Conclusions}

In this work we studied the resonance aspect of the coastal run-up as a response to incident waves. The analysis follows a normal mode approach and examines the sensitivity of those normal modes to a given linear incident wave to produce coastal run-up. These modes diverge offshore, but, since we are only interested in the coastal run-up, we can still use them. In Model-1, significant run-up sensitivity, in other words resonance, occurs only when $D$ is large. Large values of $D$ are not encountered very often in nature, not even in the shelf breaks. We also find slightly smaller runups in Model-1 if we impose a linearised seaward boundary condition (see also Antuono and Brocchini, 2007). In the two-dimensional open ocean and finite-width sloping channel case (Model-2), resonance occurs when the aspect ratio of the bay (width / length) is small. This kind of bay (or channel) geometry exists in many coastal regions such as the Tokyo Bay, making the results relevant in engineering practice.

The residue method developed here can actually be generalized for more complicated channel geometries (such as piecewise constant slopes with varying width) by performing a "fusion" of this method with the boundary elements technique. This is because the boundary elements technique has recently proven to be very efficient for solving the Helmholtz equation in multiple dimensions (Gumerov and Duraiswami, 2008; Takahashi and Hamada, 2009). Therefore, the fusion should work as follows: for a series of complex frequen- 


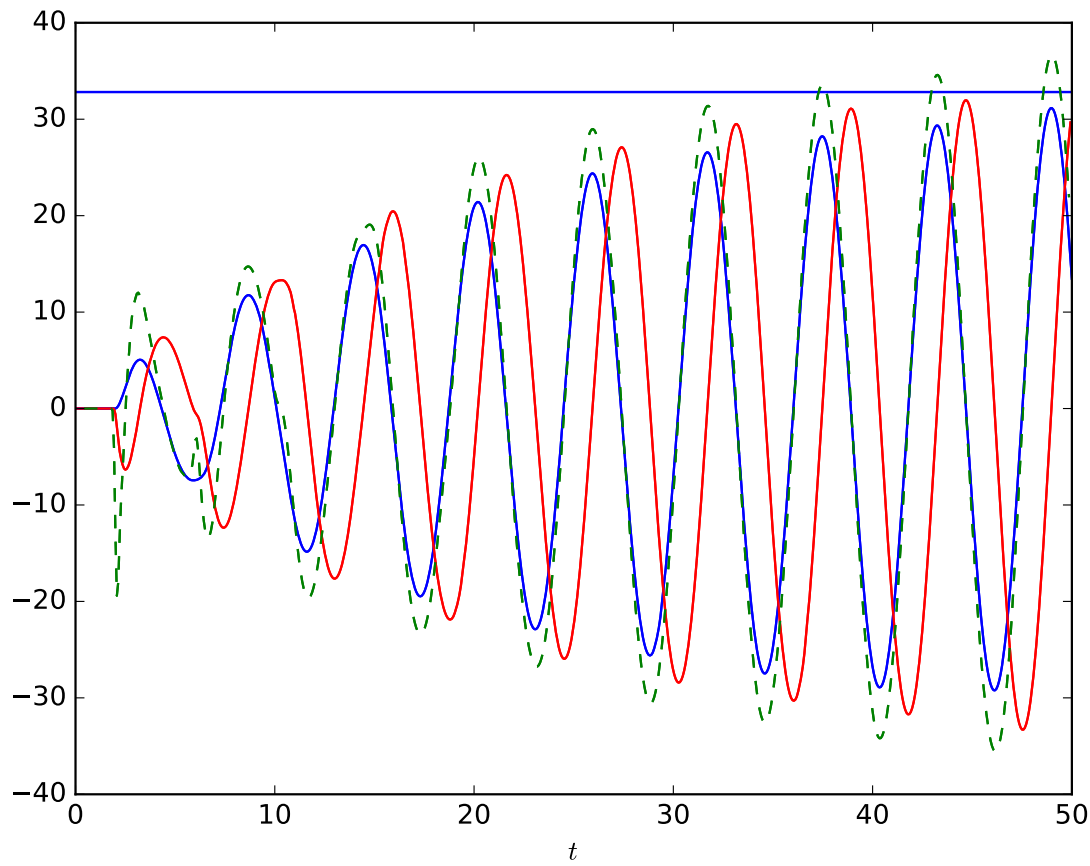

Figure 7. The blue continuous curve is the run-up (calculated using Eq. 47) normalized by the incident wave amplitude as a function of time. The channel width, $2 a$, is equal to 0.2 . The incident wave is given as $\eta_{0}^{I} \sin \left(\Re \omega_{1}(t+(x-1))\right) \theta(t+(x-1))$, where $\omega_{1}$ is the lowest free mode frequency for Model-2 (see Table 2 for $D=0$ ), smoothed by multiplying it with the tanh function as in Fig. 5. The continuous red curve is the shoreline velocity. The dashed green curve is the shoreline acceleration. The horizontal blue line is the maximum run-up calculated using conformal mapping.
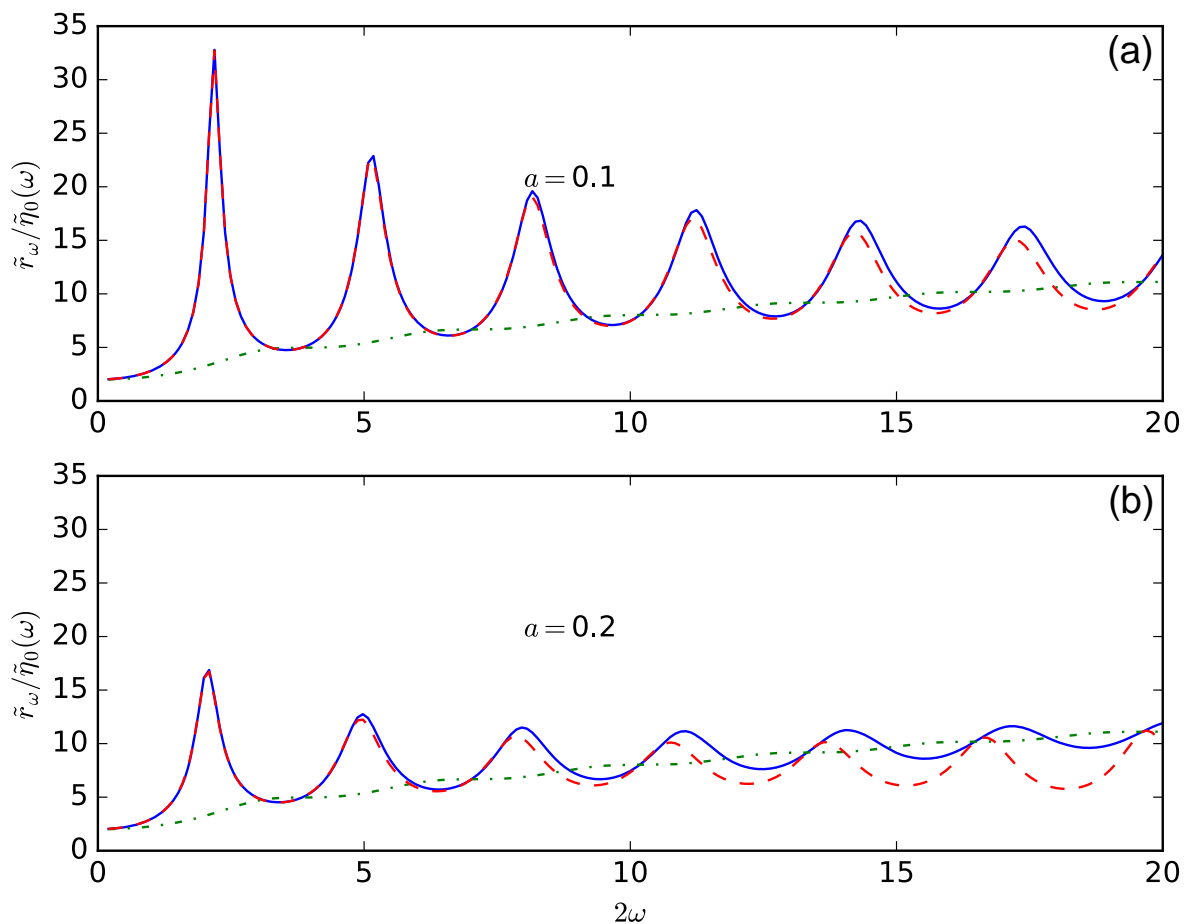

Figure 8. The maximum run-up normalized to the amplitude of the incident wave for the standing wave case for two different channel half-width values. The continuous blue curves are computed using the integral equation, and the red broken curves are obtained from the conformal mapping (see Eq. 48). The green dotted-dashed curve is the maximum run-up for the infinitely wide channel. In panel (a) the half-width of the sloping channel is 0.1 , and in panel (b) the half-width is 0.2 . 
cies, the boundary elements determine those frequencies for which the response diverges (for a single run, the boundary elements, formulated in the temporal Fourier domain, can only calculate the response for a monochromatic incident wave). These are the normal mode frequencies for the particular geometry chosen. Then for any incident wave train (this can be, for instance, buoy data time series for an incoming storm surge) we can calculate the contour integrals in Eq. (47) to compute the response much faster than inverse Fourier transforming the boundary element results. The operational fast Fourier transform-based models that use buoy data for tsunami warning (such as Lin et al., 2014) can not assimilate the buoy data continuously as it comes; they instead use the time series after a substantial part of the tsunami has passed across the buoy and then input this time series as the wave forcing to calculate run-up. This computational economisation has two advantages; the first is that these contour integrals can be pre-calculated and stored before, so during an operational emergency the only data that is needed is the wave train, and the response can be calculated very fast (the last integral in Eq. 47). The other advantage is that the number of points necessary to numerically evaluate the contour integral is very low compared to the inverse Fourier transformation along the real axis.

Data availability. No data sets were used in this article. 
Appendix A: Asymptotic approximation of the frequencies of the free modes for large $D$ in Model-1

In this appendix, the natural frequencies of Model-1 will be evaluated for large $D$ using an asymptotic approximation. The matrix relation that relates the incident wave to the waves over the slope and the reflected wave is given as

$$
\begin{aligned}
& \left(\begin{array}{cc}
J_{0}(2 \omega) & -1 / i \omega \\
-\omega J_{1}(2 \omega) & \sqrt{D+1}
\end{array}\right)\left(\begin{array}{c}
A_{0}(\omega) \\
R(\omega)
\end{array}\right)= \\
& \left(\begin{array}{c}
\widetilde{\eta}^{I}(\omega) / i \omega \\
\sqrt{D+1} \widetilde{\eta}^{I}(\omega)
\end{array}\right) .
\end{aligned}
$$

The determinant of the matrix becomes zero if $i \sqrt{D+1} J_{0}(2 \omega)-J_{1}(2 \omega)$ vanishes. Expanding natural frequencies in powers of $\varepsilon=1 / \sqrt{D+1}$, we obtain

$\omega_{k}=\omega_{k}^{(0)}+\Delta \omega_{k}=\omega_{k}^{(0)}+\varepsilon \omega_{k}^{(1)}+\varepsilon^{2} \omega_{k}^{(2)}+\ldots$,

where $\omega_{k}^{(0)}$ is the unperturbed root with $J_{0}\left(2 \omega_{k}^{(0)}\right)=0$. Up to the second order we obtain

$\omega_{k}^{(1)}=i / 2$,

$\omega_{k}^{(2)}=\frac{1}{4} \frac{J_{1}{ }^{\prime}\left(2 \omega_{k}^{(0)}\right)}{J_{0}{ }^{\prime}\left(2 \omega_{k}^{(0)}\right)}$,

where the primes mean the derivative with respect to the argument, $2 \omega_{k}^{(0)}$. Consequently,

$2 \omega_{k} \approx 2 \omega_{k}^{(0)}+\frac{i}{\sqrt{D+1}}+\frac{1}{2(D+1)} \frac{J_{1}^{\prime}\left(2 \omega_{k}^{(0)}\right)}{J_{0}^{\prime}\left(2 \omega_{k}^{(0)}\right)}$

is obtained for twice the perturbed normal mode frequencies.

\section{Appendix B: Nonlinear effects at the toe of the slope}

The solution we obtained in Eq. (16) satisfies nonlinear shallow water equations over the slope; however, it does not satisfy any nonlinearity as far as the boundary conditions at $x=1$ are concerned. In this section, we will call the potential associated with this solution $\varphi^{(0)}$, which is given as

$\varphi^{(0)}(t, x)=\left|A_{0}\right| J_{0}(2 \omega \sqrt{x+\eta}) \cos (\omega(t-u))$.

Note that this expression does not give information on the transients; it is only valid as $t \rightarrow \infty$. An incoming wave arriving from far away, considered in this work, would probably be highly linear, because otherwise it would have lost its monochromaticity due to nonlinear effects. However, near the resonant frequencies the amplitudes of oscillations over the slope (even the deeper part further offshore) are generally much larger than the amplitude of the incident wave. Nonlinear effects might therefore be important over the entire slope, including the toe region. On the toe, the upper bound of the free surface oscillations cannot be larger than twice the incident wave amplitude. However, the derivative with respect to $x$ of $\eta$ can be very large because of the discontinuity of $u$ for large $D$ and a theoretical upper limit on it cannot be found. Therefore, the linearisation of the boundary conditions might be a serious compromise.

Our aim is to calculate an additional term, $\varphi^{(1)}$, such that

$\varphi=\varphi^{(0)}+\varphi^{(1)}$

will satisfy the boundary condition at the toe, to the order $\left|A_{0}\right|^{2}$. The linear approximation to $\varphi^{(0)}$ is given as

$\varphi_{\operatorname{lin}}^{(0)}=\left|A_{0}\right| J_{0}(2 \omega \sqrt{x}) \cos (\omega t)$,

by which one can find the linear expressions of the free surface and the fluid velocity. The term that is responsible for the nonlinear correction of the boundary condition at $x=1$ is the difference between the $\varphi^{(0)}(t, x)$ and $\varphi_{\text {lin }}^{(0)}(t, x)$ near the toe of the slope, denoted as $\Delta \varphi^{(0)}$. To the first order, this is simply obtained through expanding $\varphi^{(0)}(t, x)$ around $x=1$ and $t$ :

$$
\begin{aligned}
& \Delta \varphi^{(0)} \approx\left|A_{0}\right|^{2} \omega^{2} J_{1}(2 \omega) J_{0}(2 \omega \sqrt{x}) \sin (\omega t) \cos (\omega t) \\
& +\left|A_{0}\right|^{2} \omega^{2} J_{0}(2 \omega) \frac{J_{1}(2 \omega \sqrt{x})}{\sqrt{x}} \cos (\omega t) \sin (\omega t) \\
& \quad \text { for } \quad x \rightarrow 1^{-}
\end{aligned}
$$

and $\Delta \varphi^{(0)} \equiv 0$ for $x>1$. We denote the free surface associated with $\Delta \varphi^{(1)}$ as $\eta^{(1)}$. Since $\eta^{(0)}$ associated with $\Delta \varphi^{(0)}$ has a discontinuity of the order $\left|A_{0}\right|^{2}$ at the toe, this discontinuity is remedied by adding $\eta^{(1)}=\partial_{t} \varphi^{(1)}$. The boundary condition at the toe then reads

$$
\begin{aligned}
& \left.\eta^{(1)}\right|_{x=1^{-}}-\left.\eta^{(1)}\right|_{x=1^{+}}= \\
& \left|A_{0}\right|^{2} \omega^{2}\left(-2 \omega J_{1}(2 \omega) J_{0}(2 \omega) \cos (2 \omega t)\right. \\
& \left.+\frac{1}{4} J_{1}^{2}(2 \omega)(\cos (2 \omega t)+1)\right) .
\end{aligned}
$$

For the velocities, a similar approach based on the continuity of the flux (this time taking into account the real depth, given by $1+\eta$ ) gives

$$
\begin{aligned}
& \left.u^{(1)}\right|_{x=1^{-}}-\left.(D+1) u^{(1)}\right|_{x=1^{+}}= \\
& \frac{1}{2}\left|A_{0}\right|^{2} \omega^{2}\left(-\omega J_{1}^{2}(2 \omega)+\left.J_{0}(2 \omega) \frac{\mathrm{d}}{\mathrm{d} x} J_{1}(2 \omega x)\right|_{x=1}\right. \\
& \left.\quad+\frac{3}{2} J_{0}(2 \omega) J_{1}(2 \omega)\right) \sin (2 \omega t),
\end{aligned}
$$

where $\left.\Delta u^{(0)}\right|_{x=1^{-}}=-\left.\partial_{x} \Delta \varphi^{(0)}\right|_{x=1^{-}}$and $u^{(1)}=-\partial_{x} \varphi^{(1)}$. 
Let us now propose a general solution for $\eta^{(1)}$, composed of time-dependent and time-independent parts of the form:

$$
\eta^{(1)}=\left\{\begin{array}{l}
N^{(1)} \cos (2 \omega t) J_{0}(4 \omega \sqrt{x})+C^{(1)} \\
\text { for } x<1 \\
R^{(1)} \cos \left(2 \omega t-\frac{(x-1)}{\sqrt{D+1}}\right)+\Theta^{(1)} \theta\left(t-\frac{x-1}{\sqrt{D+1}}\right) \\
\text { for } x>1 .
\end{array}\right.
$$

Here, $N^{(1)}$ is given as

$$
\begin{aligned}
& N^{(1)}= \\
& \frac{1}{2}\left|A_{0}\right|^{2} \omega^{2} \frac{\left(-\omega J_{1}^{2}(2 \omega)+\left.J_{0}(2 \omega) \frac{\mathrm{d}}{\mathrm{d} x} J_{1}(2 \omega x)\right|_{x=1}+\frac{3}{2} J_{0}(2 \omega) J_{1}(2 \omega)\right)}{J_{1}(4 \omega)-\sqrt{D+1} J_{0}(4 \omega)} \\
& -\left|A_{0}\right|^{2} \omega^{2} \frac{\left(-2 \omega J_{1}(2 \omega) J_{0}(2 \omega)+\frac{1}{4} J_{1}^{2}(2 \omega)\right) \sqrt{D+1}}{J_{1}(4 \omega)-\sqrt{D+1} J_{0}(4 \omega)}
\end{aligned}
$$

Strong resonance is expected for large values of $D$ and for $J_{0}(2 \omega) \approx 0$. Therefore, the relation for $N^{(1)}$ simplifies to $\left|A_{0}\right|^{2} \omega^{2} J_{1}^{2}(2 \omega) /\left(4 J_{0}(4 \omega)\right)$. Furthermore, we find $C^{(1)}=$ $\left|A_{0}\right|^{2} \omega^{2} J_{1}^{2}(2 \omega) / 4$ and $\Theta=0$.

\section{Appendix C: Conformal mapping for Model-2}

The problem of an incident wave into a rectangular bay of uniform depth was solved in Mei et al. (2004), page 218. We will extend their solution to the case of the sloping channel using the same conformal mapping. Actually, Kajiura (1977) also mentions a conformal mapping, but its mathematical details are not included in his paper or elsewhere in publicly available literature.

The flow displays a complex pattern in the vicinity of the mouth of the channel (see Fig. C1). If the depth discontinuity $D$ is zero, the relative change of depth in this region is small. Therefore, the linearised shallow water equation for a given frequency $\omega$ can be approximated by the Helmholtz equation $\left(\nabla^{2} \varphi+\omega^{2} \varphi=0\right)$ in this region. If the wavelength is much larger than the width of the channel $(2 a)$, then $\nabla^{2} \varphi$ is of the order of $\varphi / a^{2}$ with $1 / a^{2} \gg \omega^{2}$. Consequently, the Laplace equation can be used as an accurate approximation of the Helmholtz equation. Unlike the Helmholtz equation, however, the Laplace equation is invariant under conformal transformation. The semi-infinite channel connected to an infinite sea shown in Fig. C1 can be mapped onto the upper complex plane by conformal transformation $f(z)$. The inverse of this function is given by

$f^{-1}(\widetilde{z})=1+\frac{2 a}{\pi}\left[-i \sqrt{\widetilde{z}^{2}-1}+\ln \left(\frac{\widetilde{z}}{\sqrt{\tilde{z}^{2}-1}+i}\right)\right]$,

where $\widetilde{z}=\tilde{x}+i \widetilde{y}$ spans the upper half complex plane. The square root function used in this conformal mapping has a branch cut along the positive real axis with $\sqrt{|\widetilde{z}| \pm 0^{+} i}=$ $\pm \sqrt{|\widetilde{z}|}$. Accordingly, $f^{-1}$ maps the segment $\zeta+i 0^{+}$for $1>\zeta>0$ on one side of the semi-infinite channel, and the segment with $0>\zeta>-1$ is mapped on the other side.

The no-flux condition is also invariant under the conformal mapping and it becomes

$\partial_{\tilde{y}} \varphi\left(\tilde{x}, \tilde{y}=0^{+}\right)=0$ for $\tilde{x} \neq 0$

in $\widetilde{z}$ space. Note that the $\widetilde{z}=0$ is the singular point of $f^{-1}$. A general solution of the Laplace equation that satisfies the no-flux condition is given by

$\varphi(\widetilde{x}, \widetilde{y})=m \ln (|\widetilde{z}|)+c$,

where the coefficients $m, c$ together with coefficients $A_{0}, \widetilde{S}_{0}$ will be calculated in a way to insure a smooth transition from the solution of the Laplace equation to the inner solution, $\left(A_{0} J_{0}(2 \omega \sigma)\right)$, and to the outer solution given by Eq. (40).

For small $|\widetilde{z}|, f^{-1}$ can be approximated by

$1+\frac{2 a}{\pi}(\ln (\tilde{z} /(2 i)))=x+i y$.

Therefore, the solution of the Laplace equation, $m \ln (|\widetilde{z}|)+c$, in terms of $z=x+i y$ becomes

$m \frac{\pi(x-1)}{2 a}-m \ln (e / 2)+c$.

Matching this with the inner solution $A_{0}(\omega) J_{0}(2 \omega \sqrt{x}) \approx$ $A_{0}\left(j_{0}(2 \omega)+(x-1) J_{1}(2 \omega)\right)$ at $x=1^{-}$(note that $x=1^{-}$ means that the distance to the mouth is much smaller than the channel length but much larger than $a$, which is the width of the channel), we obtain

$A_{0}(\omega) J_{0}(2 \omega)=-m \ln (e / 2)+c$

and

$-A_{0}(\omega) \omega J_{1}(2 \omega)=\frac{m \pi}{2 a}$.

The outer solution given by Eq. (40) is, at intermediate range (that is the distance from the mouth of the channel is much larger than the width of the channel but much smaller than the wavelength), given as

$$
\frac{2 \widetilde{\eta}_{0}^{I}(\omega)}{i \omega}+\frac{2 a \widetilde{S}_{0}}{\pi} \ln \left(\frac{\Gamma}{2} \sqrt{(x-1)^{2}+y^{2}}\right) .
$$

In obtaining Eq. (C8), we made the approximation that $a \ll \sqrt{(x-1)^{2}+y^{2}}$; therefore, the Hankel function term in Eq. (40) can be taken out of the integral and approximated using the logarithm, again assuming that $\omega \ll \sqrt{(x-1)^{2}+y^{2}}$. For $|\widetilde{z}| \rightarrow \infty$, in the upper complex plane $f^{-1}(\widetilde{z})$, can be approximated by

$x+i y \approx 1-\frac{2 a i}{\pi} \widetilde{z}$. 


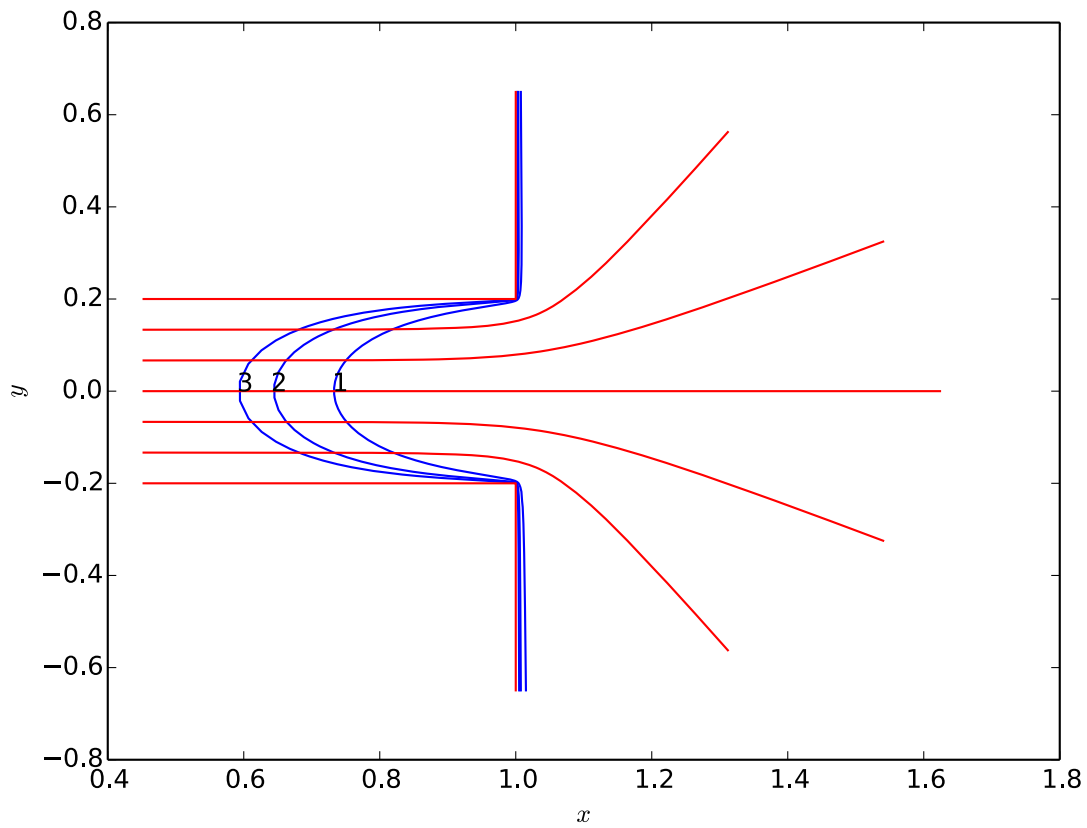

Figure C1. The blue curves are $x+i y=z=f^{-1}\left(z=\zeta+\epsilon_{n} i\right)$ for $\zeta$ varying between -5 and 5 and $\epsilon_{n}=0.06 / n$. The three blue curves correspond to $n=1,2,3$. The smaller the parameter $\epsilon_{n}$ becomes, the further left these blue curves will reach (the left extremities of these curves are approximately $\frac{2 a}{\pi} \ln \left(\epsilon_{n}\right)$ where $a$ is 0.2$)$. The red curves are the streamlines.

Therefore, the solution of the Laplace equation in the open sea is then

$$
\begin{aligned}
& m \ln \left(\frac{i \pi}{2 a} \sqrt{(x-1)^{2}+y^{2}}\right)+c \\
& \text { for }\left(\sqrt{(x-1)^{2}+y^{2}}\right) \gg a .
\end{aligned}
$$

Matching Eq. (C10) with Eq. (C8) will become

$$
\begin{aligned}
& \frac{2 a \widetilde{S}_{0}(\omega)}{\pi}=m \\
& a \widetilde{S}_{0} i+\frac{2 a \widetilde{S}_{0}}{\pi} \ln \left(\frac{\Gamma \omega}{2}\right)+\frac{2 \widetilde{\eta}^{I}(\omega)}{i \omega}=m \ln \left(\frac{\pi}{2 a}\right)+c .
\end{aligned}
$$

Solving Eqs. (C6), (C7), (C11) and (C12),

$$
\begin{gathered}
A_{0}(\omega)=\frac{2 \widetilde{\eta}^{I}(\omega)}{i \omega}\left[J_{0}(2 \omega)+a \omega J_{1}(2 \omega)\right. \\
\left.\left(\frac{-2}{\pi} \ln \left(\frac{e \pi}{2 \Gamma \omega a}\right)+i\right)\right]^{-1}
\end{gathered}
$$

is found. 
Competing interests. The authors declare that they have no conflict of interest.

Edited by: Ira Didenkulova

Reviewed by: Takenori Shimozono and three anonymous referees

\section{References}

Abcah, N., Ezersky, A., and Pelinovsky, E.: Physical modeling of resonance phenomena in the long wave dynamics, La Houille Blanche, 1, 58-65, https://doi.org/10.1051/lhb/2016008, 2016.

Antuono, M. and Brocchini, M.: The Boundary Value Problem for the Nonlinear Shallow Water Equations, Stud. Appl. Math., 119, 73-93, https://doi.org/10.1111/j.1365-2966.2007.00378.x, 2007.

Antuono, M. and Brocchini, M.: Maximum run-up, breaking conditions and dynamical forces in the swash zone: a boundary value approach, Coast. Eng., 55, 732-740, https://doi.org/10.1016/j.coastaleng.2008.02.002, 2008.

Antuono, M. and Brocchini, M.: Solving the nonlinear shallowwater equations in physical space, J. Fluid Mech., 643, 207-232, https://doi.org/10.1017/S0022112009992096, 2010.

Bowers, E. C.: Harbour resonance due to set-down beneath wave groups, J. Fluid Mech., 79, 71-92, https://doi.org/10.1017/S0022112077000044, 1977.

Brocchini, M.: The run-up of weakly-two-dimensional solitary pulses, Nonlin. Processes Geophys., 5, 27-38, https://doi.org/10.5194/npg-5-27-1998, 1998.

Brocchini, M. and Gentile, R.: Modelling the run-up of significant wave groups, nearshore and Coastal Oceanography, Cont. Shelf Res., 21, 1533-1550, https://doi.org/10.1016/S02784343(01)00015-2, 2001.

Brocchini, M. and Peregrine, D. H.: Integral flow properties of the swash zone and averaging, J. Fluid Mech., 317, 241-273, https://doi.org/10.1017/S0022112096000742, 1996.

Carrier, G. F. and Noiseux, C. F.: The reflection of obliquely incident tsunamis, J. Fluid Mech, 133, 147-160, 1983.

Carrrier, G. F., Wu, T. T., and Yeh, H.: Tsunami run-up and drawdown on a plane beach, J. Fluid Mech., 475, 77-99, 2003.

Choi, B. H., Kaistrenko, V., Kim, K. O., Min, B. I., and Pelinovsky, E.: Rapid forecasting of tsunami runup heights from 2-D numerical simulations, Nat. Hazards Earth Syst. Sci., 11, 707-714, https://doi.org/10.5194/nhess-11-707-2011, 2011.

Didenkulova, I., Pelinovsky, E., and Soomere, T.: Runup Characteristics of Symmetrical Solitary Tsunami Waves of "Unknown" Shapes, in: Tsunami Science Four Years after the 2004 Indian Ocean Tsunami: Part I: Modelling and Hazard Assessment, Birkhäuser Basel, Basel, Switzerland, 2249-2264, https://doi.org/10.1007/978-3-0346-0057-6_13, 2009.

Ezersky, A., Abcha, N., and Pelinovsky, E.: Physical simulation of resonant wave run-up on a beach, Nonlin. Processes Geophys., 20, 35-40, https://doi.org/10.5194/npg-20-35-2013, 2013a.

Ezersky, A., Tiguercha, D., and Pelinovsky, E.: Resonance phenomena at the long wave run-up on the coast, Nat. Hazards Earth Syst. Sci., 13, 2745-2752, https://doi.org/10.5194/nhess13-2745-2013, 2013b.

Fuentes, M. A., Ruiz, J. A., and Riquelme, S.: The runup on a multilinear sloping beach model, Geophys. J. Int., 201, 915-928, 2015.
Gumerov, N. and Duraiswami, R.: A broadband fast multipole accelerated boundary element method for the three dimensional Helmholtz equation, J. Acoust. Soc. Am., 125, 191-205, 2008.

Kajiura, K.: Local behaviour of tsunamis, Springer Berlin Heidelberg, Berlin, Heidelberg, Germany, 72-79, https://doi.org/10.1007/3540082530_137, 1977.

Kânoğlu, U.: Nonlinear evolution runup-rundown of long waves over sloping beach, J. Fluid Mech., 513, 363-372, 2005.

Kânoğlu, U. and Synokalis, C. E.: Initial value problem solution of non-linear shallow water-wave equations, Phys. Rev. Lett., 97, 148501, https://doi.org/10.1103/PhysRevLett.97.148501, 2005.

Kataoka, K., Hinata, H., and Nihei, Y.: Numerical estimation of inflow flux of floating natural macro-debris into Tokyo Bay, Estuarine, Coast. Shelf Sci., 134, 69-79, https://doi.org/10.1016/j.ecss.2013.09.005, 2013.

Lin, J.-H., Cheng, C.-Y., Yu, J.-L., Chen, Y.-Y., and Chen, G.-Y.: Quick Estimation Of Tsunami Induced Runup On Coastal Area, Coastal Engineering Proceedings, 1, https://doi.org/10.9753/icce.v34.currents.8, 2014.

Longuet-Higgins, M. S.: On the trapping of wave energy round islands, J. Fluid Mech., 29, 4781-4821, 1967.

Madsen, P. A. and Schäffer, H. A.: Analytical solutions for tsunami runup on a plane beach: single waves, $\mathrm{N}$-waves and transient waves, J. Fluid Mech., 645, 27-57, 2010.

Mei, C. C., Stiassnie, M., and Yue, D. K. P.: Theory and applications of ocean surface waves, Word Scientific pubishing Co Pte Ltd, Singapore, 2004.

Özeren, M. S. and Postacioglu, N.: Nonlinear landslide tsunami runup, J. Fluid Mech., 691, 440-460, 2012.

Pelinovsky, E. and Mazova, R.: Exact analytical solutions of nonlinear problems of tsunami wave run-up on slopes with different profiles, Nat. Hazards, 6, 227-249, 1992.

Press, W. H., Teukolsky, S. A., Vetterling, W. T., and Flannery, B. P.: Numerical recipes. The art of scientific computing, Cambridge University Press, New York, USA, 2007.

Stefanakis, T. S., Dias, F., and Dutykh, D.: Local Run-Up Amplification by Resonant Wave Interaction, Phys. Rev. Lett., 107, 124502, https://doi.org/10.1103/PhysRevLett.107.124502, 2011.

Stefanakis, T. S., Xu, S., Dutykh, D., and Dias, F.: Run-up amplification of transient long waves, Q. Appl. Math., 73, 177-199, 2015.

Synolakis, C. E.: The runup of solitary waves, J. Fluid Mech., 185, 523-545, 1987.

Synolakis, C. E.: On the roots of $f(z)=J_{0}(z)-i J_{1}(z)$, Q. Appl. Math., 46, 105-108, 1988.

Takahashi, T. and Hamada, T.: GPU-accelerated boundary element method for Helmholtz' equation in three dimensions, Int. J. Numer. Meth. Eng., 80, 1295-1321, https://doi.org/10.1002/nme.2661, 2009.

Volker, R., Yamazaki, Y., and Cheung, K. F.: Resonance and impact of the 2009 Samoa tsunami around Tutuila, American Samoa, Geophys. Res. Lett., 37, L21604, https://doi.org/10.1029/2010GL044419, 2010.

Yamazaki, Y. and Cheung, K. F.: Shelf resonance and impact of near-field tsunami generated by the 2010 Chile earthquake, Geophys. Res. Lett., 38, L12605, https://doi.org/10.1029/2011GL047508, 2011.

Zhang, J. E. and Wu, T. Y.: Oblique long waves on beach and induced longshore current, J. Eng. Mech., 125, 812-826, 1999. 